\title{
Spatio-Temporal Join Selectivity
}

Jimeng Sun

Department of Computer Science Carnegie Mellon University

Pittsburgh, PA, USA

jimeng@cs.cmu.edu

Dimitris Papadias

Department of Computer Science

Hong Kong University of Science and Technology

Clear Water Bay, Hong Kong

dimitris@cs.ust.hk

\author{
Yufei Tao \\ Department of Computer Science \\ City University of Hong Kong \\ Tat Chee Avenue, Hong Kong \\ taoyf@cs.cityu.edu.hk \\ George Kollios \\ Department of Computer Science \\ Boston University \\ Boston, MA, USA \\ gkollios@cs.bu.edu
}

\begin{abstract}
Given two sets $S_{1}, S_{2}$ of moving objects, a future timestamp $t_{q}$, and a distance threshold $d$, a spatio-temporal join retrieves all pairs of objects that are within distance $d$ at $t_{q}$. The selectivity of a join equals the number of retrieved pairs divided by the cardinality of the Cartesian product $S_{1} \times S_{2}$. This paper develops a model for spatio-temporal join selectivity estimation based on rigorous probabilistic analysis, and reveals the factors that affect the selectivity. Initially, we solve the problem for 1D (point and rectangle) objects whose location and velocities distribute uniformly, and then extend the results to multi-dimensional spaces. Finally, we deal with nonuniform distributions using a specialized spatio-temporal histogram. Extensive experiments confirm that the proposed formulae are highly accurate (average error below 10\%).
\end{abstract}

Information Systems, 31(8), 793-813, 2006.

Recommended by Yannis Ioannidis, Area Editor.

\section{Contact Author:}

Dimitris Papadias

Department of Computer Science

Hong Kong University of Science and Technology

Clear Water Bay, Hong Kong

Office: $++852-23586971 \quad$ http://www.cs.ust.hk/ dimitris/

Fax: ++852-23581477_ E-mail: dimitris@cs.ust.hk 


\section{INTRODUCTION}

An important operation in spatio-temporal databases and mobile computing systems is to predict objects' future location based on information at the current time (e.g., for collision detection). For this purpose the database usually represents object movement as a function of time and stores the function parameters [14, $1,24,23]$. As an example, given the location $o(0)$ of object $o$ at the current time 0 and its velocity $o_{V}$, its position at future time $t$ can be computed as $o(t)=o(0)+o_{V} \cdot t$. According to this representation, an update is necessary only when the function parameters (i.e., $o_{V}$ ) change.

Given two sets $S_{1}, S_{2}$ of objects, a spatio-temporal join retrieves all pairs of objects $<_{o_{1}}, o_{2}>$ such that $o_{1} \in S_{1}, o_{2} \in S_{2}$, and $\left|o_{I}\left(t_{q}\right), o_{2}\left(t_{q}\right)\right| \leq d$, i.e., the distance $\left|o_{I}\left(t_{q}\right), o_{2}\left(t_{q}\right)\right|$ between objects $o_{1}$ and $o_{2}$ at a (future) query timestamp $t_{q}$ is below a threshold $d$. For instance, consider the query "retrieve all pairs of airplanes that will come closer than 10 miles 5 minutes from now". This (self-join) example outputs pairs of moving objects; in some cases one of the inputs can be static: "retrieve all pairs $<$ city $c$, typhoon $t>$ such that $t$ will cover $c$ at 9am tomorrow according to the current spreading speed of $t$ '. An important variation is the constrained join, which involves an additional constraint window to limit the data space of interest. For instance, in the previous example, an analyst may be interested only in cities in the southeast US region.

In this paper we discuss the selectivity of spatio-temporal joins, which is defined as the number of result pairs divided by the size of the cartesian product of the input datasets. Estimating the join selectivity is important for several reasons.

- As with conventional and spatial [16] databases, selectivity estimation is vital to query optimization for computing the best execution plan.

- In many applications users are interested in the number of joined pairs (i.e., a join counting query) rather than the concrete results. For example, prediction of potential congestions requires the traffic volume rather than the ids of cars [21]. Furthermore, stream (spatio-temporal) databases [11,8] may maintain only aggregate information in order to deal with voluminous updates.

- Performing an exact join (which is time consuming) is meaningless in applications with very frequent motion function changes because the result may already be invalidated before the join processing terminates. In such cases, a fast estimation of the output size is the only meaningful computation that can be performed given the tight time limit.

Although spatial join selectivity can be computed using several methods $[4,9,22]$, their application in spatio-temporal scenarios leads to significant error. Similarly, existing work [7] on estimation of spatiotemporal window queries on a single dataset cannot be efficiently adapted for joins. Motivated by this, we 
address the problem by first proposing fundamental probabilistic formulae for spatio-temporal joins on uniform (point and rectangular) objects. Then, we integrate these equations with spatio-temporal histograms to support non-uniform data. Compared to the previous approaches, the proposed histogram achieves significantly lower estimation error and is incrementally updatable (whereas previous solutions require frequent re-construction). We evaluate the efficiency of our methods with extensive experimentation.

The rest of the paper is organized as follows. Section 2 reviews previous work on spatial joins, histograms, and spatio-temporal prediction. Section 3 analyzes spatio-temporal join selectivity on uniform data, while Section 4 extends the results to non-uniform data (using histograms). Section 5 experimentally evaluates the proposed methods, and Section 6 concludes the paper with directions for future work.

\section{RELATED WORK}

Section 2.1 overviews spatial join algorithms (assuming knowledge of R-trees [10,6]), and join selectivity estimation on static objects. Section 2.2 introduces MinSkew, a multi-dimensional histogram that constitutes the starting point of our spatio-temporal histogram. Section 2.3 discusses spatio-temporal range selectivity and elaborates why it cannot be applied for join selectivity estimation. Finally Section 2.4 reviews the Time Parameterized R-tree (TPR-tree), an index structure for moving objects which is employed in our histogram techniques.

\subsection{Spatial Join Selectivity}

Consider two objects $o_{1}, o_{2}$ belonging to spatial datasets $S_{1}, S_{2}$ and satisfying the join condition $\left|o_{1}, o_{2}\right| \leq d$. Join processing algorithms $[5,12,15]$ are based on the observation that $o_{1}$ and $o_{2}$ should reside in R-tree nodes $E_{1}$ and $E_{2}$ respectively, whose minimum bounding rectangles (MBRs) satisfy the property $\mid E_{l}$, $E_{2} \mid \leq d$. Thus, qualifying pairs are retrieved by synchronously traversing the R-trees of $S_{1}$ and $S_{2}$ in a topdown fashion, recursively following node pairs that are within the distance constraint. The special case where $d=0$ corresponds to the intersection join and has received considerable attention. Notice, however, that intersection join is not meaningful for point data since it reduces to equality of points.

Spatial join selectivity was first studied in [3], which presents a formula for uniform data using the results of previous work $[13,20]$ on window query selectivity. Several histogram-based approaches have been proposed for non-uniform distributions. In particular, the histograms of [27, 16] divide the universe regularly, while more sophisticated techniques $[4,22]$ perform the partitioning according to the data distribution. On the other hand [9] employs a different approach based on power laws. Further, [16] studies the selectivity of complex queries that involve multiple datasets. All the above methods require the knowledge of distributions of the join datasets. In spatio-temporal databases, however, the distribution 
continuously changes due to object movements. Hence, it is extremely expensive (both in terms of computation time and storage cost) to pre-compute the distributions for future timestamps. Furthermore, even if such distributions are obtained, they will soon be invalidated due to subsequent updates, rendering re-computation necessary. Therefore, traditional approaches for spatial join selectivity are insufficient for moving objects. Effective techniques should take the specialized problem characteristics into account.

\subsection{MinSkew}

MinSkew [2] is a spatial histogram originally proposed for selectivity estimation of window queries in non-uniform datasets. It partitions the space into a set of buckets such that the MBRs of all buckets are disjoint, and their union covers the entire universe. Each bucket $b_{i}$ contains the number $b_{i}$.num of objects whose centroids fall inside $b_{i}$.MBR, and the average extent $b_{i}$.len of these objects. Figure 1 illustrates a query $q$ and an intersecting bucket $b$ in the $2 \mathrm{D}$ space. The gray area corresponds to the intersection between $b$.MBR and the extended query region, obtained by enlarging each edge of $q$ with distance b.len/2. The expected number of rectangles in $b$ intersecting $q$ is estimated as b.num $\times$ areaG/area(b.MBR), where area $G$ and area(b.MBR) are the areas of the gray region and $b$. MBR respectively. The estimated selectivity is obtained by summing the results for all such intersecting buckets.

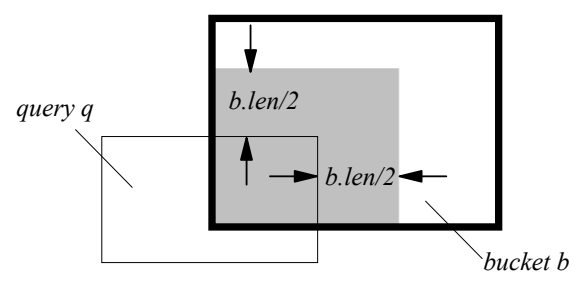

Figure 1: Estimating the selectivity inside a bucket

To ensure satisfactory accuracy, the above estimation requires that objects in each bucket $b$ have similar sizes and their centroids distribute uniformly in b.MBR. To quantify the degree of uniformity, [2] defines the spatial-skew (denoted as b.skew) for a bucket $b$ as the variance ${ }^{1}$ of the spatial densities ${ }^{2}$ of all points inside it. Since a small spatial-skew indicates better uniformity, MinSkew aims at minimizing $\sum_{i=1 \sim n}\left(b_{i} \cdot n u m \cdot b_{i}\right.$. skew $)$, i.e., the weighted sum of the spatial-skews of the buckets. Computing the optimal buckets, however, is NP-hard [17]. To reduce the computation cost, [2] partitions the original space into a grid with $H \times H$ regular cells (where $H$ is a parameter called the resolution), and associates each cell $c$ with (i) the number c.num of objects whose centroids fall in c.MBR, (ii) the average extent length c.len of objects satisfying (i), and (iii) the density $c . d e n$ of the cell (i.e., the number of objects intersecting $c . M B R$ ).

\footnotetext{
${ }^{1}$ Given $n$ numbers $a_{1}, a_{2}, \ldots, a_{n}$, the variance equals $\frac{1}{n} \sum_{i=1}^{n}\left(a_{i}-\bar{a}\right)^{2}$, where $\bar{a}$ is the average of $a_{1}, a_{2}, \ldots, a_{n}$.

2 The density of a point is defined as the number of objects that cover the point.
} 
Figure 2a shows an example $(H=3)$ for a dataset with 8 objects, and Figure $2 \mathrm{~b}$ illustrates the information associated with the cells (len is not shown because it is not needed for partitioning). A greedy algorithm builds the histogram that minimizes the total-skew, under the constraint that each bucket must cover an integer number of cells. The final buckets are shown in Figure 2c, together with their associated information (notice that the spatial skews are very small) computed as follows:

$$
\begin{gathered}
\text { b.num }=\sum_{\text {each cell } \text { in } b} \text { c.num }, \\
\text { b.len }=\frac{\sum_{\text {each cell } \text { in } b} \text { c.num } \cdot \text { c.len }}{\sum_{\text {each cell c in } b} \text { c.num }}, \\
\text { b.skew }=\frac{1}{|C|} \sum_{\text {each cell } \text { in } b}(c . d e n-\overline{d e n})^{2}
\end{gathered}
$$

where $|C|$ is the number of cells covered by $b$, and $\overline{d e n}$ denotes their average density. MinSkew can be applied in arbitrary dimensionality with straightforward modifications.

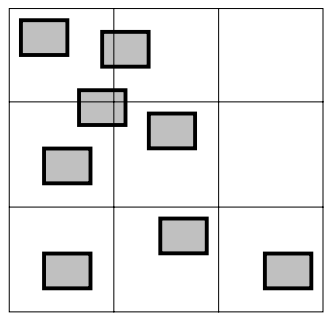

(a) $3 \times 3$ grid

\begin{tabular}{|c|c|c|}
\hline $\begin{array}{c}\text { num=1 } \\
D=3\end{array}$ & $\begin{array}{c}\text { num=1 } \\
D=2\end{array}$ & $\begin{array}{c}\text { num=0 } \\
D=0\end{array}$ \\
\hline $\begin{array}{c}\text { num=2 } \\
D=2\end{array}$ & $\begin{array}{c}\text { num=1 } \\
D=2\end{array}$ & $\begin{array}{c}\text { num=0 } \\
D=0\end{array}$ \\
$\begin{array}{c}\text { num=1 } \\
D=1\end{array}$ & $\begin{array}{c}\text { num=1 } \\
D=1\end{array}$ & $\begin{array}{c}\text { num=1 } \\
D=1\end{array}$ \\
\hline
\end{tabular}

(b) Cell information

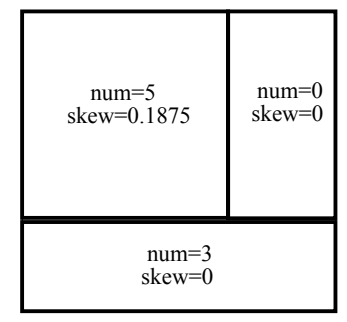

(c) The final buckets

Figure 2: Building the histogram

\subsection{Spatio-Temporal Range Selectivity}

Given a set of moving objects, a spatio-temporal window query retrieves all the objects that appear in a query region at the query time $t_{q}$. Choi and Chung [7] extend MinSkew with velocities to estimate the selectivity of window queries (defined as the ratio between the number of qualifying objects and the dataset cardinality). Figure 3a shows 8 moving points, where the arrows (numbers) indicate the directions (values) of their velocities (a minus value indicates movement in the negative direction of the axis). Figure $3 \mathrm{~b}$ shows the corresponding spatio-temporal histogram built in two steps. First, the spatial extents of the buckets are determined in the same way as the traditional MinSkew algorithm (by ignoring the velocities). Then, each bucket $b$ is associated with a velocity bounding rectangle (VBR) $\left(b_{V x-}, b_{V x+}, b_{V y-}\right.$, $\left.b_{V y+}\right)$, such that (i) $b_{V x^{-}}\left(b_{V x^{+}}\right)$equals the minimum (maximum) velocity along the $\mathrm{x}$-dimension of the objects inside, and (ii) $b_{V y-}\left(b_{V y^{+}}\right)$is defined similarly with respect to the y-dimension. In Figure $3 \mathrm{~b}$, the VBRs of buckets $b_{1}, b_{2}$, and $b_{3}$ are $(-2,1,-3,1),(0,0,0,0)$, and $(-1,2,-1,-1)$ respectively. 
Accurate spatio-temporal selectivity estimation requires that the location (velocities) of the objects inside a bucket uniformly distribute(s) in the bucket's MBR (VBR). The data partition in Figure 3, however, is decided according to spatial information; thus, the uniformity of velocity cannot be guaranteed, which may lead to significant estimation error. Furthermore, the histogram is not incrementally updatable and must be re-built very frequently to maintain satisfactory accuracy ([7] suggests re-building at every single timestamp). To see this, assume that in Figure $3 b$ the y-velocity of object $d$ (which determines $b_{1 V y}$-) changes to -1, after which $b_{1 V y \text { - }}$ should be adjusted to the y-velocity of $c$ (i.e., -2), because it is now the minimum y-velocity of all objects in $b_{1}$. This, however, is not possible because the histogram does not contain detailed information about the velocities of individual objects.

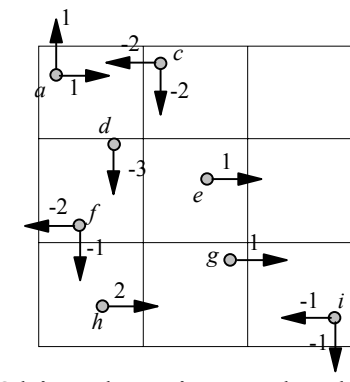

(a) Object location and velocities

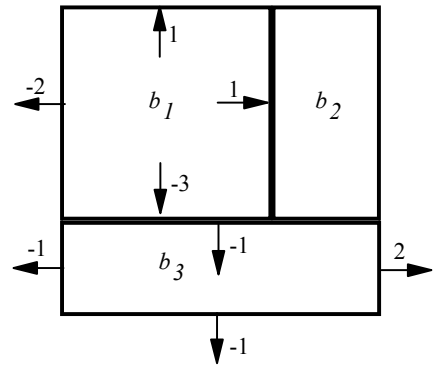

(b) The final buckets

Figure 3: A spatio-temporal histogram

Based on the above histogram, Choi and Chung [7] discuss selectivity estimation for (predictive) range queries on point data. Specifically, given a point $q$, distance $d$, and timestamp $t_{q}$, a range query retrieves all the objects whose distances from $q$ at time $t_{q}$ are less than $d$. The selectivity is defined as the number of retrieved objects divided by the dataset cardinality. This technique could be applied to estimate join selectivity (on point datasets) by regarding a join as the combination of multiple range queries. Specifically, given two datasets $S_{1}, S_{2}$, we build a histogram on $S_{1}$. Then, for each point $p_{2} \in S_{2}$, the number of objects $p_{1}$ in $S_{I}$ that qualify the join condition with $p_{2}$ (i.e., $\left.\left|p_{I}\left(t_{q}\right), p_{2}\left(t_{q}\right)\right| \leq d\right)$ can be predicted using the method in [7]. The total number of qualifying pairs equals the sum of the estimates for all points in $S_{2}$. This approach, however, has the following problems. First, it incurs significant computation overhead because, the number of range selectivity predictions equals the cardinality of $S_{2}$ (which can be huge in practice). Second, it requires maintaining all the objects in one dataset, leading to expensive space consumption. Further, for large datasets, some data might need to be stored on disk, in which case selectivity estimation would involve I/O accesses, further compromising the estimation time. In Section 3 , we develop alternative solutions to overcome these problems. 


\subsection{The TPR-Tree}

The TPR-tree [24] is an extension of the R-tree that can answer predictive queries on moving objects. Each object is represented with an MBR that bounds its extents at the current time, and a velocity vector. Figure 4a shows the representation of two objects $u$ and $v$. As before, the arrows indicate the velocity directions, and the numbers correspond to their values. A non-leaf entry (e.g., $E$ in Figure $4 a$ ) stores an MBR and a VBR. Specifically, as in traditional R-trees the MBR tightly encloses the extents of all entries in the node at the current time, while the definition of VBR is similar to those of the spatio-temporal buckets in Figure 4b (i.e., it tightly bounds the velocities of the objects in its subtree). The MBR of an entry $E$ continuously grows with time according to its VBR, which ensures that the MBR always bounds the underlying objects (but is not necessarily tight).

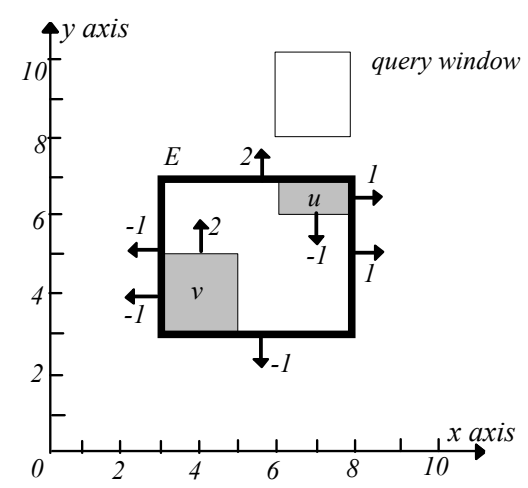

(a) The boundaries at current time 0

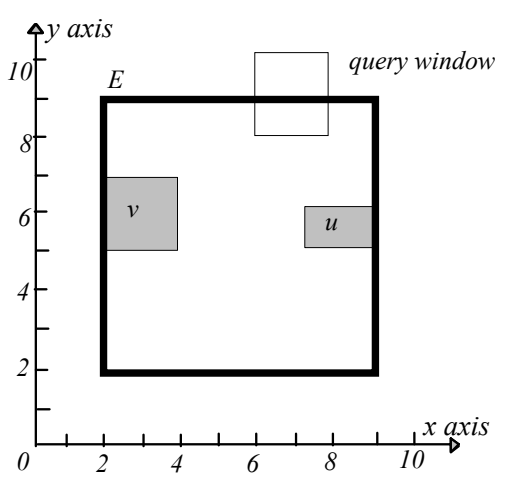

(b) The boundaries at future time 1

Figure 4: Representation of entries in the TPR-tree

Figure $4 \mathrm{~b}$ shows $u, v$ and the enclosing node $E$ at time 1 . Observe how the extents and positions of $u, v, E$ change, and that the rectangle of $E$ is larger than the tightest MBR for $u$ and $v$. Future MBRs (e.g., those in Figure 4b) are not stored explicitly, but are computed based on the entries' current extents and velocities. A spatio-temporal window query is processed in exactly the same way as in the R-tree, except that the extents of the MBRs at some future time are first calculated dynamically before being compared to the query window. For the query (retrieving objects intersecting the query window at timestamp 1) shown in Figure 4, for example, node $E$ must be visited because its MBR intersects the query at time 1 (although its MBR at time 0 does not).

\section{SPATIO-TEMPORAL JOIN SELECTIVITY}

Formally, given two sets $S_{1}, S_{2}$ of $m$-dimensional moving objects (points or rectangles), a future timestamp $t_{q}$, and distance threshold $d$, a spatio-temporal join retrieves all pairs of objects $\left\langle o_{1}, o_{2}\right\rangle$ such that $o_{1} \in S_{1}, o_{2} \in S_{2}$, and $\left|o_{I}\left(t_{q}\right), o_{2}\left(t_{q}\right)\right| \leq d$, where $\left|o_{I}\left(t_{q}\right), o_{2}\left(t_{q}\right)\right|$ is the distance between $o_{1}$ and $o_{2}$ at $t_{q}$. We consider that the distance $\left|o_{I}\left(t_{q}\right), o_{2}\left(t_{q}\right)\right|$ is computed according to the $L_{p}$ norm. Specifically, denoting the 
coordinates of an $m$-dimensional point $p$ as $p . x_{0}, p . x_{1}, \ldots, p . x_{m}$, the $L_{p}$-distance $\left|o_{l}\left(t_{q}\right), o_{2}\left(t_{q}\right)\right|$ equals $\left(\sum_{i=1 \sim d}\left|p_{1} \cdot x_{i}-p_{2} \cdot x_{i}\right|^{p}\right)^{1 / p}$. A constrained spatio-temporal join is similar to a normal spatio-temporal join, except that it involves a constraint window $W_{q}$ which is an $m$-dimensional rectangle. A pair of qualifying objects $<o_{1}, o_{2}>$ must satisfy all the conditions of a normal join, and the additional predicate that $o_{1}$ and $o_{2}$ both intersect $W_{q}$ at time $t_{q}$. The selectivity of the (normal or constrained) join is the ratio between the number of result pairs and the size of the Cartesian product $S_{1} \times S_{2}$.

Interestingly, as discussed in [9], the join selectivity under various $L_{p}$ norms (for different $p$ ) differs only by a constant factor. As a result, to deal with arbitrary $L_{p}$ norm, it suffices to solve the problem under a particular value of $p$. In the sequel, we focus on $L_{\infty}$ (i.e., $\left.\left|o_{I}\left(t_{q}\right), o_{2}\left(t_{q}\right)\right|=\max _{i=1,2, \ldots, m}\left|p_{1} . x_{i}-p_{2} . x_{i}\right|\right)$, since the resulting equations are the simplest in this case. The distance between two (hyper-) rectangles $r_{1}, r_{2}$ is the minimum of the distances between all pairs of points in $r_{1}$ and $r_{2}$ respectively, or more formally: $\mid r_{l}$, $r_{2} \mid=\min \left\{\left|p_{1}, p_{2}\right|\right.$ for all $p_{1} \in r_{1}$, and $\left.p_{2} \in r_{2}\right\}$. Under $L_{\infty}$, two (point or rectangle) objects $o_{1}, o_{2}$ are within distance $d$ if and only if the extended region of $o_{1}$, obtained by enlarging its extents with length $d$ along all dimensions, intersects $o_{2}$. Figure 5a and b illustrate this for 2D points and rectangles, respectively. When $d=0$, the condition $\left|o_{I}\left(t_{q}\right), o_{2}\left(t_{q}\right)\right| \leq d$ reduces to simple intersection.

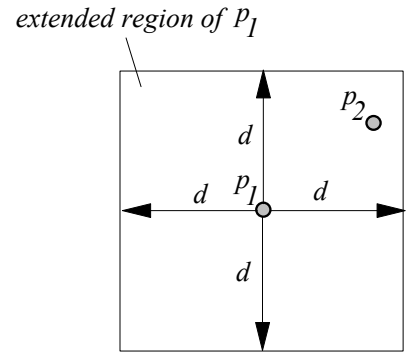

(a) Points

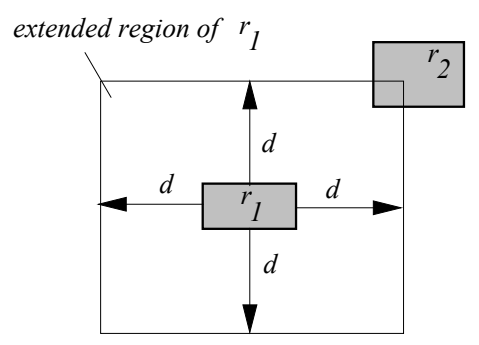

(b) Rectangles

Figure 5: Objects within distance $d$ (the $L_{\infty}$ norm) from each other

We use $R_{l}\left(V_{l}\right)$ to represent the MBR (VBR) that tightly encloses the location (velocities) of all the objects in $S_{1}$, and similarly, $R_{2}\left(V_{2}\right)$ for $S_{2}$. Note that, $R_{1}$ and $R_{2}$ may cover different sub-spaces of the universe (i.e., we allow objects of the two sets to distribute in different areas). The objective is to predict the join selectivity based solely on $R_{1}, R_{2}, V_{1}, V_{2}$, assuming that (i) the location of the objects inside $S_{I}\left(S_{2}\right)$ distributes uniformly in $R_{1}\left(R_{2}\right)$, (ii) the object velocities are uniform in $V_{I}\left(V_{2}\right)$, and (iii) all the dimensions are independent. Section 3.1 first solves the problem for one-dimensional space, and Section 3.2 extends the results to higher-dimensionality, both assuming $L_{\infty}$ Section 3.3 extends the results to the constrained join and other $L_{p}$ norms. In Section 4 we overcome the uniformity assumptions with the aid of histograms. Table 1 lists the symbols that will appear frequently in our analysis. 


\begin{tabular}{|c|c|}
\hline Symbol & Description \\
\hline$M$ & dimensionality of the data space \\
\hline$t_{q}$ & the query timestamp \\
\hline$S_{1}, S_{2}$ & The two datasets participating the join \\
\hline$R_{1}\left(R_{2}\right)$ & The MBR of $S_{1}\left(S_{2}\right)$ covering the location of objects in the set \\
\hline$V_{1}\left(V_{2}\right)$ & The VBRs of $S_{I}\left(S_{2}\right)$ covering the velocities of objects in the set \\
\hline$N_{l}\left(N_{2}\right)$ & The number of objects in $S_{1}\left(S_{2}\right)$ \\
\hline$p \cdot x_{i}$ & The $i$-th coordinate of point $p$ \\
\hline$\left[R \cdot x_{i-}, R \cdot x_{i^{+}}\right]$ & The extent along the $i$-th dimension of MBR $R$ \\
\hline$\left[V . v_{i-}, V \cdot v_{i^{+}}\right]$ & The extent along the $i$-th dimension of VBR $V$ \\
\hline$P Q_{\{1 D p t, 1 \text { Dintv }\}}$ & The qualifying probability for 1D \{point, interval\} objects \\
\hline $\operatorname{Sel}_{\{1 D p t, 1 \text { Dintv, }, \text { t, rect }\}}$ & The join selectivity for $\{1 \mathrm{D}$ points, $1 \mathrm{D}$ intervals, $m \mathrm{D}$ points, $m \mathrm{D}$ rectangles $\}$ \\
\hline$W_{q}$ & The constrained window \\
\hline
\end{tabular}

Table 1: Frequent symbols in the analysis

\subsection{Solution for One-Dimensional Space}

We first consider the case of $1 \mathrm{D}$ point data and then solve the problem for $1 \mathrm{D}$ intervals. Let $\left[R_{1} \cdot x_{-}, R_{l} \cdot x_{+}\right]$ be the extent of $R_{l}$ (the MBR of $S_{l}$ ), and $\left[V_{l} \cdot v_{-}, V_{l} \cdot v_{+}\right]$the range of $V_{l}$ (the VBR of $S_{l}$ ). The Cartesian product $S_{1} \times S_{2}$ consists of $N_{1} \cdot N_{2}$ pairs of points. Let $P Q_{1 D p t}$ be the probability for a pair to qualify the join condition; the expected number of qualifying pairs can be represented as $N_{l} \cdot N_{2} \cdot P Q_{1 D p t}$. Furthermore, since the selectivity equals the number of qualifying pairs divided by $N_{l} \cdot N_{2}, P Q_{I D p t}$ directly corresponds to the join selectivity. In particular, the derivation of $P Q_{1 D p t}$ is equivalent to the following problem: Given two points $p_{1}, p_{2}$ such that $p_{1}\left(p_{2}\right)$ uniformly distributes in the range $\left[R_{1} \cdot x_{-}, R_{1} \cdot x_{+}\right]\left(\left[R_{2} \cdot x_{-}, R_{2} \cdot x_{+}\right]\right)$, and the velocity of $p_{I}\left(p_{2}\right)$ uniformly distributes in $\left[V_{l} \cdot v_{-}, V_{l} \cdot v_{+}\right]\left(\left[V_{2} \cdot v_{-}, V_{2} \cdot v_{+}\right]\right)$, compute the probability $P Q_{1 D p t}$ that $\left|p_{I}\left(t_{q}\right), p_{2}\left(t_{q}\right)\right| \leq d$.

For two particular points $p_{1}, p_{2}$, the probability for $\left\langle p_{1}, p_{2}\right\rangle$ to qualify depends on their relative positions. To see this, consider Figure 6a, where the $\mathrm{x}$ - and y-axes correspond to the spatial and temporal dimensions respectively (the current time is 0 ). The thick line segments (on the spatial dimension) represent the MBRs of $S_{1}$ and $S_{2}$, while their VBRs are illustrated by the lines passing through the end points of the corresponding MBRs (the slopes of these lines indicate the velocity values). At query time $t_{q}$, the entire range that can be reached by $p_{l}$ constitutes line segment $A B$, where point $A(B)$ is the extreme position if $p_{1}$ travels with the minimum (maximum) velocity $V_{l} \cdot v_{-}\left(V_{l} \cdot v_{+}\right)$. The probability that $p_{l}$ reaches any point on segment $A B$ is the same, due to the fact that the velocity of $p_{1}$ uniformly distributes in the range $\left[V_{l} . v_{-}, V_{l} \cdot v_{+}\right]$(note that: every position on segment $A B$ can be reached via a unique velocity of $p_{l}$ ). Similarly, segment $C D$ consists of all the possible location of $p_{2}$ at time $t_{q}$. Assuming the distance threshold $d$ is $0,<p_{1}, p_{2}>$ may qualify because, as shown in Figure 6a, segments $A B$ and $C D$ intersect (i.e., it is possible for $p_{1}$ and $p_{2}$ to reach the same position at $t_{q}$ ). 


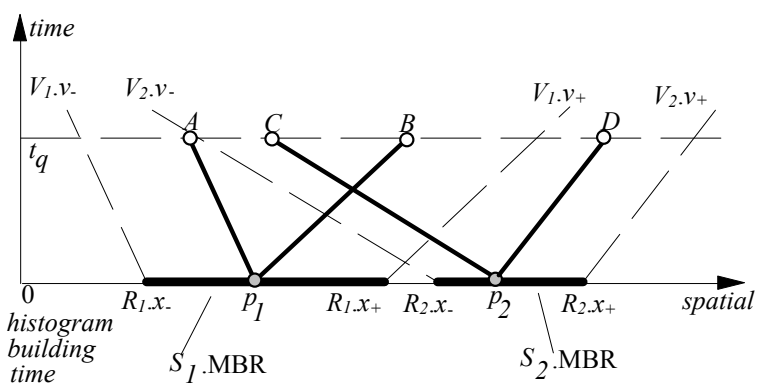

(a) $<p_{1}, p_{2}>$ may qualify

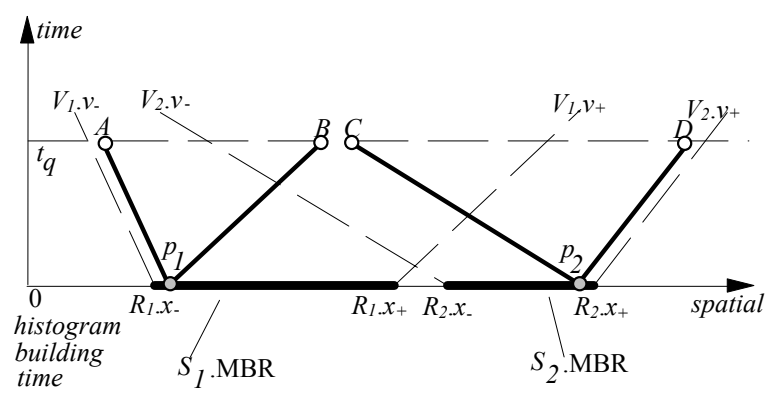

(b) $<p_{1}, p_{2}>$ cannot qualify

Figure 6: Qualifying pairs (distance threshold $d=0$ )

Figure $6 \mathrm{~b}$ shows similar situation except that $p_{1}$ and $p_{2}$ are farther apart from each other at the current time. Notice that, in this case $A B$ and $C D$ do not intersect, indicating that $\left\langle p_{1}, p_{2}\right\rangle$ does not belong to the result. Motivated by this, we denote with $P_{\text {pair }}\left(l_{1}, l_{2}\right)$ the probability that pair $\left\langle p_{1}, p_{2}>\right.$ qualifies if $p_{1}\left(p_{2}\right)$ lies at location $l_{1}\left(l_{2}\right)$. Consequently, the qualifying probability $P Q_{1 D p t}$ corresponds to the average of $P_{\text {pair }}\left(l_{1}, l_{2}\right)$ over all possible positions for $l_{1}$ and $l_{2}$, or formally:

$$
P Q_{1 D p t}=\frac{1}{\left(R_{1} \cdot x_{+}-R_{1} \cdot x_{-}\right)\left(R_{2} \cdot x_{+}-R_{2} \cdot x_{-}\right)} \int_{R_{1} \cdot x_{-}}^{R_{1} \cdot x_{+} \cdot x_{-} \cdot x_{-} \cdot x_{+}} P_{\text {pair }}\left(l_{1}, l_{2}\right) d l_{2} d l_{1}
$$

Given two points at $l_{1}$ and $l_{2}$ respectively, $P_{\text {pair }}\left(l_{1}, l_{2}\right)$ corresponds to $\mathrm{P}\left\{\left|p_{I}\left(t_{q}\right), p_{2}\left(t_{q}\right)\right| \leq d\right\}$, i.e., the probability that the distance of $p_{1}$ and $p_{2}$ at time $t_{q}$ is not greater than the threshold $d$. Assuming the velocities of $p_{1}$ and $p_{2}$ to be $u_{1}$ and $u_{2}$ respectively, $p_{l}\left(t_{q}\right)$ and $p_{2}\left(t_{q}\right)$ can be represented as:

$$
p_{1}\left(t_{q}\right)=l_{1}+t_{q} \cdot u_{1} \text {, and } p_{2}\left(t_{q}\right)=l_{2}+t_{q} \cdot u_{2}
$$

Thus, $P_{\text {pair }}\left(l_{1}, l_{2}\right)=P\left\{\left|p_{1}\left(t_{q}\right), p_{2}\left(t_{q}\right)\right| \leq d \mid p_{1}=l_{1}\right.$ and $\left.p_{2}=l_{2}\right\}=P\left\{\left|\left(l_{1}-l_{2}\right)+t_{q} \cdot\left(u_{1}-u_{2}\right)\right| \leq d\right\}$

The above equation can be converted to:

$$
P_{p a i r}\left(l_{1}, l_{2}\right)=P\left\{\frac{l_{1}-l_{2}-d}{t_{q}}+u_{1} \leq u_{2} \leq \frac{l_{1}-l_{2}+d}{t_{q}}+u_{1}\right\}
$$

Since $u_{1}$ and $u_{2}$ uniformly distribute in $\left[V_{1} . v_{-}, V_{1} \cdot v_{+}\right]$and $\left[V_{2} . v_{-}, V_{2} . v_{+}\right]$respectively, they satisfy the following probability density functions:

$$
f\left(u_{1}\right)=\frac{1}{V_{1} \cdot v_{+}-V_{1} \cdot v_{-}} \text {, and } f\left(u_{2}\right)=\frac{1}{V_{2} \cdot v_{+}-V_{2} \cdot v_{-}}
$$

Therefore, the right hand side of equation 2 can be written s $^{3}$ :

\footnotetext{
${ }^{3}$ In this paper we follow the convention that if $a>b$, then $\int_{a}^{b} f(x) d x=0$.
} 


$$
\begin{gathered}
P\left\{\frac{l_{1}-l_{2}-d}{t_{q}}+u_{1} \leq u_{2} \leq \frac{l_{1}-l_{2}+d}{t_{q}}+u_{1}\right\}=\int_{V_{1} \cdot v_{-}}^{V_{1} \cdot v_{+}}\left\{f\left(u_{1}\right)\left[\int_{\max \left(V_{2} \cdot v_{-}, u_{1}+\left(l_{1}-l_{2}-d\right) / t_{q}\right)}^{\min \left(V_{2} \cdot v_{+}, u_{1}+\left(_{1}-l_{2}+d\right) / t_{q}\right)} f\left(u_{2}\right) d u_{2}\right]\right\} d u_{1} \\
=\frac{1}{\left(V_{1} \cdot v_{+}-V_{1} \cdot v_{-}\right)\left(V_{2} \cdot v_{+}-V_{2} \cdot v_{-}\right)} \int_{V_{1} \cdot v_{-}}^{V_{1} \cdot v_{+}} \int_{\max \left(V_{2} \cdot v_{-}, u_{1}+\left(l_{1}-l_{2}-d\right) / t_{q}\right)}^{\min \left(V_{2} \cdot v_{+}, u_{1}+\left(l_{1}-l_{2}+d\right) / t_{q}\right)} 1 d u_{2} d u_{1}
\end{gathered}
$$

The above integral can be solved into closed form as presented in the appendix. To understand the formula, consider Figure 7, where point $p_{l}\left(t_{q}\right)$ shows the location of $p_{l}$ at $t_{q}$ when it travels at speed $u_{l}$ $\left(\in\left[V_{l} . v_{-}, V_{l} . v_{+}\right]\right)$. Then, line segment $A B$ corresponds to the set of positions for a qualifying point $p_{2}$ at time $t_{q}$ (i.e., $p_{2}\left(t_{q}\right)$ is within distance $d$ from $\left.p_{l}\left(t_{q}\right)\right)$.

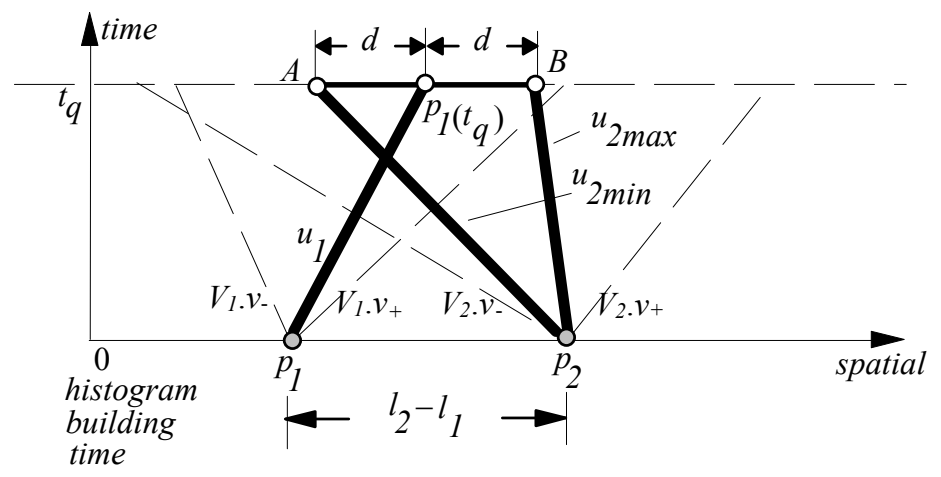

Figure 7: Computing the probability $P_{\text {pair }}\left(l_{1}, l_{2}\right)$

Let $u_{2 \min }$ and $u_{2 \max }$ be the velocities according to which $p_{2}$ reaches points $A$ and $B$ at time $t_{q}$ respectively. It follows that the probability that $\left\langle p_{1}, p_{2}>\right.$ is a join result, is $\left(u_{2 \max }-u_{2 \min }\right) /\left(V_{2} \cdot v_{+}-V_{2} \cdot v_{-}\right)$, i.e., the probability that the velocity $u_{2}$ of $p_{2}$ falls in the range $\left[u_{2 \min }, u_{2 \max }\right]$, given that $u_{2}$ uniformly distributes in $\left[V_{2} \cdot v_{-}, V_{2} \cdot v_{+}\right]$. So far we have considered a particular value of $u_{l}$, while, as shown in equation 3 , in order to compute $P_{\text {pair }}\left(l_{1}, l_{2}\right)$ we must consider all possible values in $\left[V_{l} \cdot v_{-}, V_{l} . v_{+}\right]$(i.e., the outer integral in the formula). Combining equations $1,-2,-3$, we have represented $P Q_{1 D p t}$ as a function of $R_{1}, R_{2}, V_{1}, V_{2}$; the following equation gives the complete formula for join selectivity of $1 \mathrm{D}$ points:

$$
\begin{aligned}
& \operatorname{Sel}_{1 D-p t}\left(d, t_{q}\right)= \\
& \frac{1}{\left(R_{1} \cdot x_{+}-R_{1} \cdot x_{-}\right)\left(R_{2} \cdot x_{+}-R_{2} \cdot x_{-}\right)\left(V_{1} \cdot v_{+}-V_{1} \cdot v_{-}\right)\left(V_{2} \cdot v_{+}-V_{2} \cdot v_{-}\right)} \int_{R_{1} \cdot x_{-}}^{R_{1} \cdot x_{+} \cdot R_{2} \cdot x_{-} \cdot x_{+} V_{+} \cdot V_{1} \cdot v_{+}} \int_{\max }^{\min \left(V_{2} \cdot v_{+}, u_{1}+\right.} \int_{\left.\left(V_{2} \cdot v_{-}, u_{1}+l_{1}-l_{2}+d\right) / t_{q}\right)}^{\left.\left(l_{1}-l_{2}-d\right) / t_{q}\right)} 1 d u_{2} d u_{1} d l_{2} d l_{1}
\end{aligned}
$$

Extending the results to interval objects is straightforward. Assume that $S_{1}\left(S_{2}\right)$ contains intervals with lengths $I_{1}\left(I_{2}\right)$; then, similar to the discussion for point objects, the selectivity corresponds to the 
qualifying probability $P Q_{1 \text { Dintv }}$ that a pair of intervals $<i_{1}, i_{2}>$ in the cartesian product $S_{1} \times S_{2}$ has distance no longer than $d$ at future time $t_{q}$. Observe that $i_{1}$ and $i_{2}$ are closer than $d$, if and only if their centroids are within distance $d+\left(I_{1}+I_{1}\right) / 2$. This is illustrated in Figure 8, where intervals $i_{1}$ and $i_{2}$ (with lengths $I_{1}$ and $I_{2}$ ) travel at velocities $u_{1}$ and $u_{2}$ respectively. Therefore, the selectivity $P Q_{I \text { Dintv }}$ can be estimated using equation 4, except that, as shown in equation 5, (i) $d$ should be replaced with $d+\left(I_{I}+I_{I}\right) / 2$, and (ii) the lower/upper limit of the integral should be modified to capture the fact that the centroid of $i_{l}$ distributes in $\left[R_{1} \cdot x_{-}+I_{1} / 2, R_{1} \cdot x_{+}-I_{1} / 2\right]$ (the range is $\left[R_{2} \cdot x_{-}+I_{2} / 2, R_{2} \cdot x_{+}-I_{2} / 2\right]$ for $\left.i_{2}\right)$.

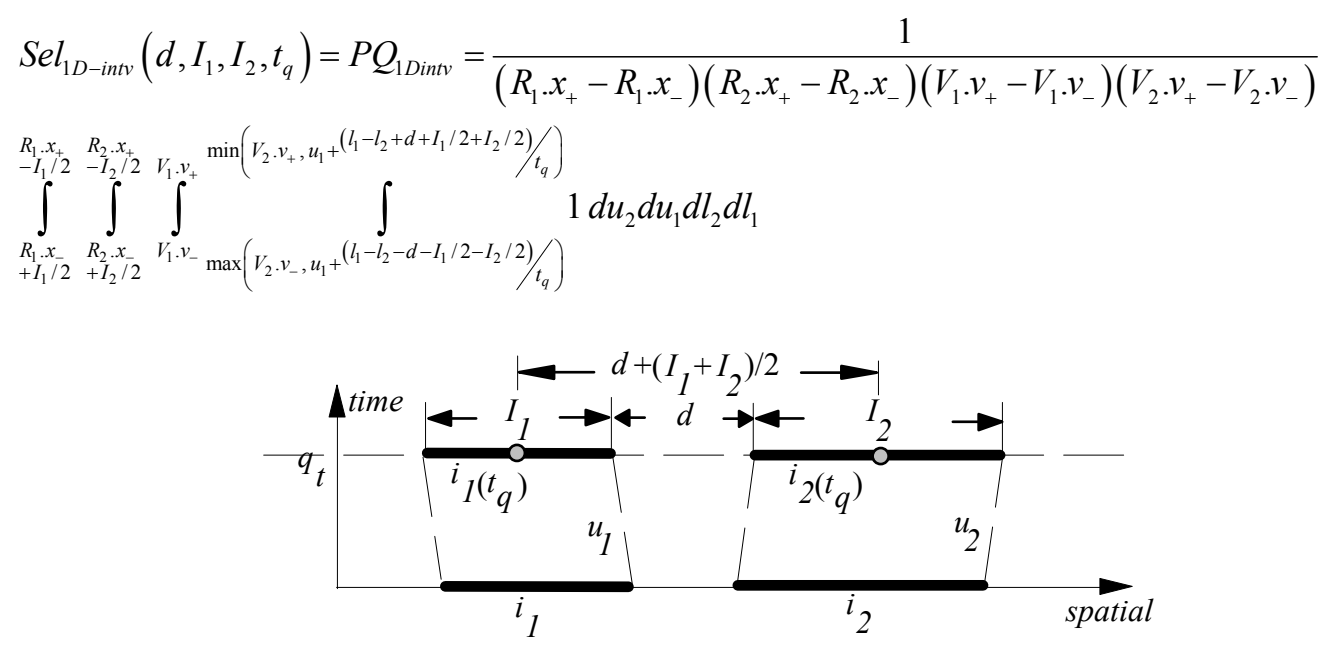

Figure 8: A pair of qualifying intervals

\subsection{Arbitrary Dimensionality}

In this section we present the equations for spatio-temporal join selectivity in $m$-dimensional spaces, starting with point datasets before extending the results to rectangles. The MBR $R_{l}\left(\mathrm{VBR} V_{l}\right)$ of set $S_{l}$ is now an $m$-dimensional rectangle, and its extent along the $i$-th dimension $(1 \leq i \leq m)$ is $\left[R_{1} \cdot x_{i-}, R_{1} \cdot x_{i+}\right]\left(\left[V_{l} \cdot v_{i-}\right.\right.$, $\left.V_{1} . v_{i+}\right]$ ); similar notations are used for $S_{2}$. Let $P Q_{p t}$ be the probability that a pair of points $\left\langle p_{1}, p_{2}>\right.$ in $S_{1} \times S_{2}$ satisfies the join condition. The crucial observation is that (due to the definition of $\left.L_{\infty}\right)\left|p_{1}\left(t_{q}\right), p_{2}\left(t_{q}\right)\right| \leq d$ if and only if $\left|p_{1} \cdot x_{i}\left(t_{q}\right)-p_{2} \cdot x_{i}\left(t_{q}\right)\right| \leq d$ for all dimensions $1 \leq i \leq m$, where $p_{1} \cdot x_{i}\left(t_{q}\right)$ represents the $i$-th coordinate of $p_{1}$ at time $t_{q}$. Let $P Q_{I D p t-i}(1 \leq i \leq m)$ be the probability that the $i$ th coordinates of $p_{1}$ and $p_{2}$ qualify. Since the dimensions are independent, we have:

$$
P Q_{p t}=\prod_{i=1}^{m} P Q_{1 D p t-i}
$$

The computation of $P Q_{1 D p t-i}$ along each dimension is based on equation 4 (passing $d, t_{q}$ ), except that $R_{l} x_{-}$, $R_{l} \cdot x_{+}, V_{l} \cdot v_{-}, V_{l} \cdot v_{+}$should be replaced with $R_{l} \cdot x_{i-}, R_{l} \cdot x_{i+}, V_{l} \cdot v_{i-}$, and $V_{l} \cdot v_{i^{+}}$respectively. As in the $1 \mathrm{D}$ case, $P Q_{p t}$ corresponds to the selectivity $\operatorname{Sel}_{\text {point }}\left(d, t_{q}, m\right)$. 
In order to estimate the join selectivity for rectangular objects, we denote $I_{l i}\left(I_{2 i}\right)$ as the extents of the objects in $S_{l}\left(S_{2}\right)$ along the $i$-th dimension. Given a pair of rectangles $\left\langle r_{1}, r_{2}>\right.$, let their extents (on the $i$-th dimension) at time $t_{q}$ be $r_{1} . i_{i}\left(t_{q}\right)$ and $r_{2} . i_{i}\left(t_{q}\right)$, respectively. In analogy with the point case, $<r_{1}, r_{2}>$ is a join result if and only if $<r_{1} . i_{i}\left(t_{q}\right), r_{2} . i_{i}\left(t_{q}\right)>$ qualifies along the $i$ th dimension $(1 \leq i \leq m)$, or equivalently, $\mid r_{1} . i_{i}\left(t_{q}\right)$, $r_{2} . i_{i}\left(t_{q}\right) \mid \leq d$. Hence, the selectivity for $m$-dimensional rectangles is represented as:

$$
\operatorname{Sel}_{\text {rect }}\left(d, t_{q}, m\right)=\prod_{i=1}^{m} P Q_{1 \text { Dintv }-i}
$$

where $P Q_{1 \text { Dintv- } i}$ is the probability that $<r_{1} . i_{i}\left(t_{q}\right), r_{2} . i_{i}\left(t_{q}\right)>$ qualifies, and is computed according to equation 5 (passing $d, I_{1 i}, I_{2 i}$, and $t_{q}$ ).

\subsection{Extensions}

In this section, we first study the selectivity estimation for constrained joins (again for $L_{\infty}$ metric), and then explain the extension to arbitrary $L_{p}$ norms. Our analysis of the constrained join follows the same framework as the discussion on normal joins. Specifically, we first solve the fundamental problem involving 1D points, and then tackle the general version (multi-dimensional, rectangle data) by reducing it to the fundamental case. Figure 9 illustrates an example, where line segment $A B$ denotes the constrained window $W_{q}$ at time $t_{q}$ (i.e., if $<p_{1}, p_{2}>$ is a qualifying join pair, then both $p_{1}$ and $p_{2}$ must appear in $A B$ at $t_{q}$ ). Similar to the derivation in Section 3.1, we fix the location of $p_{1}$ and $p_{2}$ at time 0 to specific positions $l_{l}, l_{2}$, respectively, and the velocity of $p_{1}$ to a particular value $u_{1}$. Note that the permissible values of $u_{1}$ (in order to appear in $W_{q}$ at $t_{q}$ ) depend on the location $l_{l}$ of $p_{l}$. In Figure 9, for instance, the minimum (maximum) value $u_{1 \min }\left(u_{1 \max }\right)$ of $u_{l}$ is such that $p_{I}$ will reach point $A(B)$ (i.e. an end point of $W_{q}$ ) at this speed.

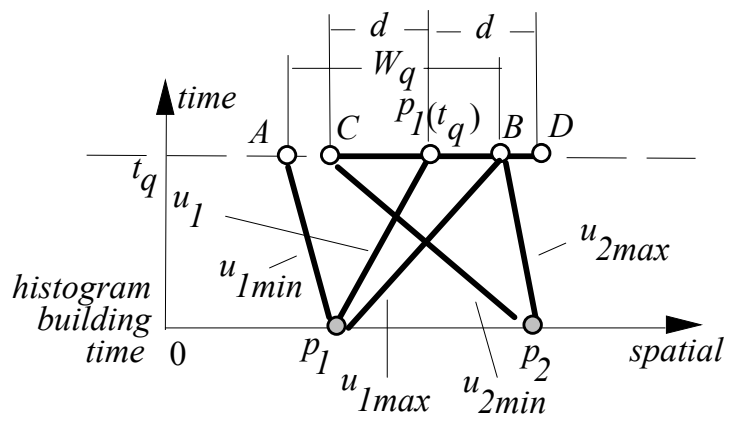

(a) Deriving $P_{C-p a i r}\left(l_{1}, l_{2}, u_{1}, W_{q}\right)$ for point data

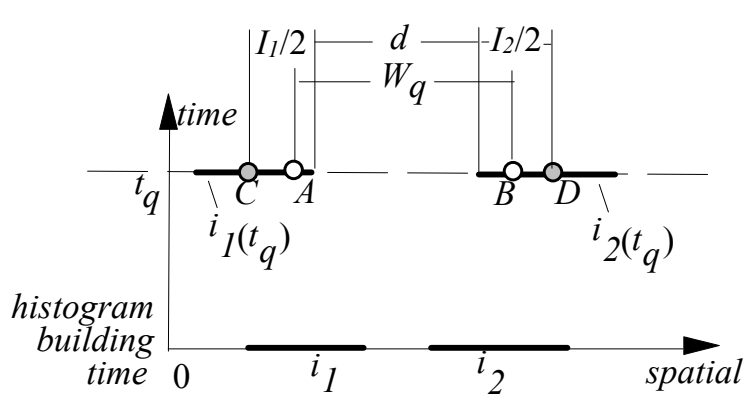

(b) A pair of qualifying intervals

Figure 9: Analysis of constrained joins

Having fixed $p_{l}\left(t_{q}\right)$, the possible location of $p_{2}\left(t_{q}\right)$ (for $\left\langle p_{1}, p_{2}\right\rangle$ to qualify the join predicate) is confined to segment $C B$, which contains all the positions that are in $W_{q}$ and have distances at most $d$ from $p_{l}\left(t_{q}\right)$. Consequently, the minimum $u_{2 \min }$ (maximum $u_{2 \max }$ ) velocity of $p_{2}$ is decided by the slope of segment 
connecting $p_{2}$ and $C(B)$. Therefore, the probability $P_{C-p a i r}\left(l_{1}, l_{2}, u_{1}, W_{q}\right)$ that $<p_{1}, p_{2}>$ qualifies (given $l_{1}, l_{2}$, $u_{1}$ ) equals $\left(\min \left(V_{2} \cdot v_{+}, u_{2 \max }\right)-\max \left(V_{2} \cdot v_{\rightarrow}, u_{2 \min }\right)\right) /\left(u_{2 \max }-u_{2 \min }\right)$, taking into account the fact that $u_{2}$ is in $\left[u_{2 \min }\right.$, $\left.u_{2 \max }\right]$. Similar to the discussion in Section 3.1, the overall probability $P_{C-1 D p t}$ (i.e., the selectivity $\operatorname{Sel}_{C-1 D p t}$ ) can be obtained by integrating $P_{C-p a i r}\left(l_{l}, l_{2}, u_{1}, W_{q}\right)$ over all the possible values for $l_{1}, l_{2}, u_{1}$, as shown in equation (8):

$$
\begin{aligned}
\operatorname{Sel}_{C-1 D p t}\left(d, t_{q}, W_{q}\right)=P_{C-1 D p t}\left(d, t_{q}, W_{q}\right) & =\frac{1}{\left(R_{1} \cdot x_{+}-R_{1} \cdot x_{-}\right)\left(R_{2} \cdot x_{+}-R_{2} \cdot x_{-}\right)\left(V_{1} \cdot v_{+}-V_{1} \cdot v_{-}\right)\left(V_{2} \cdot v_{+}-V_{2} \cdot v_{-}\right)} \\
& \int_{R_{1} \cdot x_{-}}^{R_{1} \cdot x_{+} \cdot R_{2} \cdot x_{-} \cdot x_{-}} \int_{u_{\text {min }}}^{x_{+}} \int_{u_{\max }}\left(\min \left(V_{2} \cdot v_{+}, u_{2 \max }\right)-\max \left(V_{2} \cdot v-, u_{2 \min }\right)\right) d u_{1} d l_{2} d l_{1}
\end{aligned}
$$

Next we study constrained joins on 1D intervals. Figure 9 shows two intervals $i_{1}, i_{2}$ (with lengths $I_{1}, I_{2}$, respectively) that qualify the join (note that both $i_{1}\left(t_{q}\right)$ and $i_{2}\left(t_{q}\right)$ intersect the constraint region $W_{q}$ ). The crucial observation is that, the centroids of $i_{1}, i_{2}$ must satisfy the following conditions: (i) the distance between them is within $d+\left(I_{1}+I_{2}\right) / 2$ (similar to Figure 8), and (ii) the centroid of $i_{1}\left(i_{2}\right)$ should have distance no more than $W_{q} / 2+I_{1} / 2\left(W_{q} / 2+I_{2} / 2\right)$ from the centroid of $W_{q}$ in order for $i_{l}\left(t_{q}\right)\left(i_{2}\left(t_{q}\right)\right)$ to intersect $W_{q}$. Hence, the selectivity for intervals can be reduced to equation 8 by considering the interval centroids (as with equation 5, the integral ranges must be modified to ensure that both $i_{1}$ and $i_{2}$ lie in $R_{1}$ and $R_{2}$, respectively). The extension to multiple dimensions is trivial: we simply multiply the selectivity on each individual axis, as shown in Section 3.2.

We finish this section by clarifying the application of our techniques to general $L_{p}$ norms other than $L_{\infty}$. For this purpose, we review the concept of sphere ratio $\operatorname{sr}\left(L_{p 1}, L_{p 2}, m\right)$ between two different norms $L_{p 1}$, $L_{p 2}\left(p_{1} \neq p_{2}\right)$ for dimensionality $m$. Specifically, $\operatorname{sr}\left(L_{p 1}, L_{p 2}, m\right)$ equals the ratio between the volumes of $m$ dimensional spheres with the same radius in $L_{p 1}$ and $L_{p 2}$ (it can be easily verified that the ratio is a constant independent of the radius). To illustrate this, Figure 10 demonstrates the spheres with radius $d$ under $L_{1}, L_{2}, L_{\infty}$ in the 2D space (particularly, the sphere of $L_{2}$ is simply a circle). The sphere ratio between $L_{1}\left(L_{\infty}\right)$ and $L_{2}$, for example, equals the area of the inner (outer) square divided by that of the circle. Faloutsos et al. [9] show the following interesting result: if $\operatorname{Sel}_{p 1}$ and $\operatorname{Sel}_{p 2}$ are the join selectivities under the $L_{p 1}$ and $L_{p 2}$ metrics and $m$ is the dimensionality, then $\operatorname{Sel}_{p 1} / \operatorname{Sel}_{p 2}$ equals $\operatorname{sr}\left(L_{p 1}, L_{p 2}, m\right)$. Based on this observation, they estimate the selectivity of arbitrary $L_{p}$ norm by multiplying the selectivity of $L_{\infty}$ with $\operatorname{sr}\left(L_{p 1}, L_{p 2}, m\right)$. The same method can also be applied in our case. 


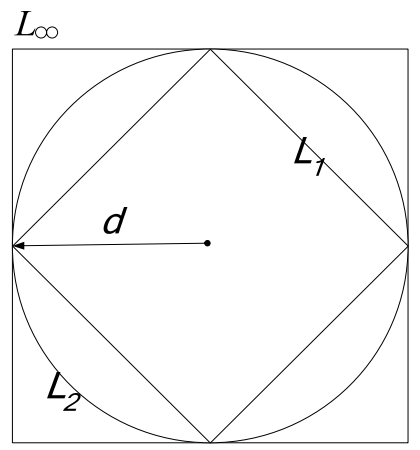

Figure 10: Spheres in $L_{1}, L_{2}, L_{\infty}$ norms

\section{SPATIO-TEMPORAL HistogRAMS}

In the previous section we presented the formulae for estimating the join selectivity for datasets where objects' location and velocities distribute uniformly in their corresponding ranges. The uniformity assumption, however, does not usually hold in real datasets, and thus direct application of the above models will lead to significant error. In this section we deal with this problem using histograms that partition the universe into separate buckets, such that the data distribution within a bucket is nearly uniform. Then, the previous equations are applied locally (in each bucket), and the overall prediction is obtained by summing up the individual estimations. The accuracy of this approach depends on the quality of the histogram, which must guarantee that in each bucket both the location and the velocity distributions are as uniform as possible. In Section 4.1, we first show that existing histograms cannot achieve this (thus, leading to erroneous estimation), and then provide an alternative solution to avoid their defects. Section 4.2 explains how to use the proposed histogram to perform estimation, as well as various approaches to reduce the computation cost.

\subsection{Histogram Construction and Maintenance}

The spatio-temporal of [7] first partitions the objects based on their spatial location using the conventional MinSkew algorithm, and then decides the VBRs of the buckets during a second step. Since the velocity information is not considered during data partition, the resulting histogram cannot ensure the uniformity of velocity distribution in the buckets. Assume, for example, that we want to build a histogram with 2 buckets for the dataset in Figure 11a. In Figure 11b the buckets are decided based on the objects' location. In particular, the first two columns of cells are grouped into the same bucket because they all contain exactly one point (i.e., no variance), while cells in the last column (with 2 points each) constitute the second bucket. Although the location distribution is fairly uniform, the velocity distribution is rather skewed. Consider the left bucket in Figure 11b, whose (x-) velocity range is $[-10,8]$ (i.e., decided by the velocities of points $a$ and $e$ ). Notice that, there are 5 points with velocities in the range [-10, -2], while 
only one (i.e., $e$ ) has positive velocity (8). Similarly, the velocity range of the right bucket is $[-8,10]$, but ranges $[-8,0]$ and $[2,10]$ contain 2 and 4 points.

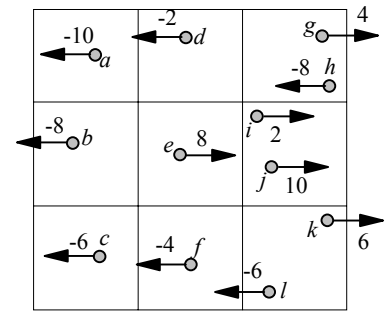

(a) Cell information

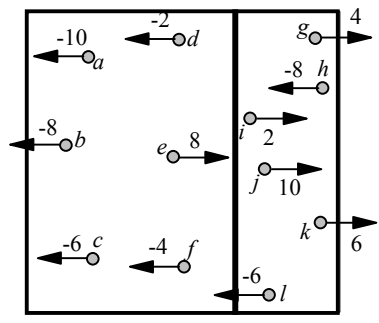

(b) Considering only location

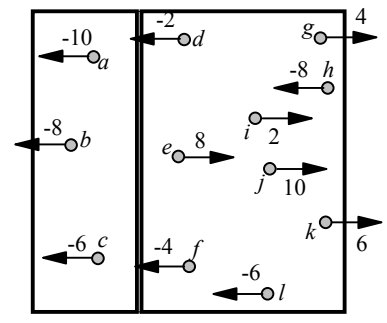

(c) Location and velocity

Figure 11: Uniform velocity distribution

An effective spatio-temporal histogram should partition data using both location and velocity information. Continuing the previous example, Figure 11c shows such a histogram, where the left and right buckets contain the first and the last two columns of cells respectively. Compared with Figure 11b, the spatial uniformity is slightly worse (only in the right bucket), while the velocity uniformity is significantly better. Specifically, the velocities distribute uniformly in the ranges $[-10,-6]$ and $[-8,10]$ for the two buckets, respectively. As a result, the new histogram is expected to produce better prediction.

The overall velocity distribution for the dataset of Figure 11 is uniform. If the distribution is skewed, ignoring the velocities during partitioning is even more problematic. Consider, for example, Figure 12a where object velocities have only two values -10 and 10. Partitioning the spatial universe is useless because (i) the overall location distribution is already fairly uniform (i.e., 2 points in each cell), and (ii) for all possible partitions, the resulting buckets still have extremely skewed velocity distribution. In fact, in this case the best partition should be based entirely on the velocity dimension. Specifically, the first bucket (Figure 12b) contains all the points with negative velocities while the second one (Figure 12c) involves those with positive ones. Notice that the resulting buckets have uniform location (one point from each cell) and velocity (all points have the same velocity) distributions.

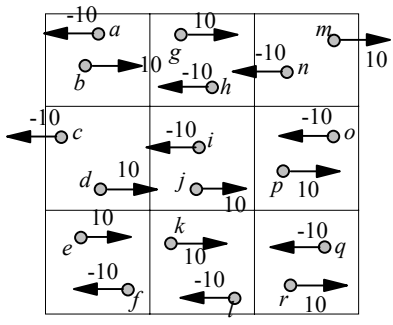

(a) Cell information

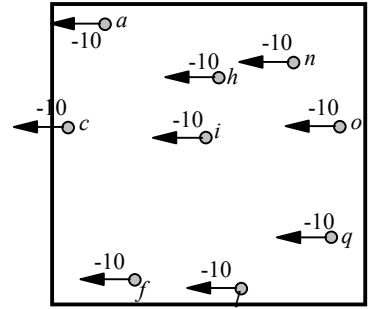

(b) Bucket 1

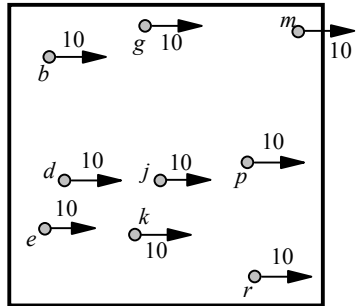

(c) Bucket 2

Figure 12: Skewed velocity distribution

Motivated by this observation, given a 2D spatial universe, we employ a histogram in the 4-dimensional space consisting of two spatial (same as the original universe) and two velocity (decided by the maximum 
and minimum velocities along the corresponding spatial axis) dimensions. Specifically, a point ( $\left.p . x_{1}, p . x_{2}\right)$ with velocities $\left(p . v_{1}, p . v_{2}\right)$ is converted into a $4 \mathrm{D}$ point $\left(p . x_{1}, p . x_{2}, p . v_{1}, p . v_{2}\right)$, and similarly a rectangle $r$ is transformed to a $4 \mathrm{D}$ one with the same extents on the spatial and velocity dimensions. The histogram is constructed using the MinSkew algorithm with an initial grid that partitions the space into $H^{4}$ regular cells. Each bucket $b$ is associated with $b$.MBR that encloses the MBRs of all the cells in $b$, and with $b$.VBR that tightly bounds the cell VBRs. For point datasets, b.num records the number of 4D points in $b$. For rectangle sets, b.num is the number of $4 \mathrm{D}$ rectangles whose centroids lie in $b$, and b.len (b.vlen) is the average spatial (velocity) extents of these rectangles. The discussion generalizes to arbitrary dimensionality in a straightforward manner.

An important property of the proposed histogram is that it can be incrementally maintained. Figure 13 illustrates this using point data with one space/velocity dimension (extending to higher dimensionality and rectangles is straightforward). Figure $13 \mathrm{a}$ and $\mathrm{b}$ illustrate the bucket extents in the space-velocity and space-time universe, respectively. Particularly, in the space-time universe, a velocity is represented as the slope of a line. Consider point $p$ which falls in bucket $b_{2}$, given its current location (as shown in both figures) and velocity (25). Assume that $p$ generates a velocity change (to -10 ) at future time $t$ (when its location is $p(t)$ ). To decide the bucket affected by $p(t)$ (in the histogram constructed at time 0 ), we find a point $p^{\prime}$ (called the surrogate point) at time 0 , such that $p^{\prime}$ will reach the same position $p(t)$ with the updated velocity (-10) of $p$. As shown in Figure 13b, $p^{\prime}$ is the intersection of the spatial axis and the line with slope -10 that crosses $p(t)$. The bucket affected by $p(t)$ is the one $\left(b_{3}\right)$ that contains $p^{\prime}$ in the spacevelocity universe, as shown in Figure 13a. Since the new bucket $b_{3}$ is different from the old one $b_{2}$, the histogram must be modified by decreasing $b_{2} . n u m$ and increasing $b_{3}$.num (by 1 ). In some cases, the surrogate point may fall outside the universe, in which case the boundary bucket needs to be enlarged. As an example, the MBR of bucket $b_{3}$ must be expanded to cover the surrogate point $q^{\prime}$ (of $q$ ) in Figure 13. It is worth mentioning that, the VBR of the selected bucket for expansion includes the updated velocity of $q^{\prime}$ (i.e., hence $b_{4}$ is not expanded).

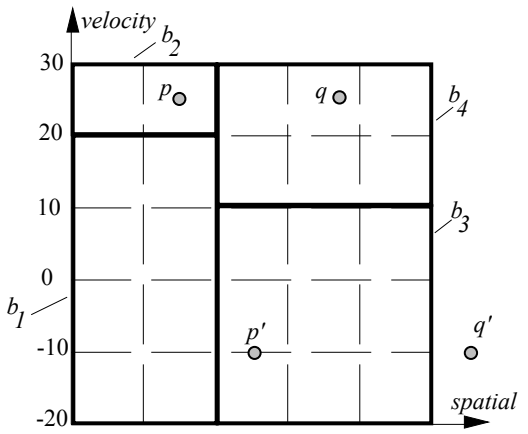

(a) The cells and buckets

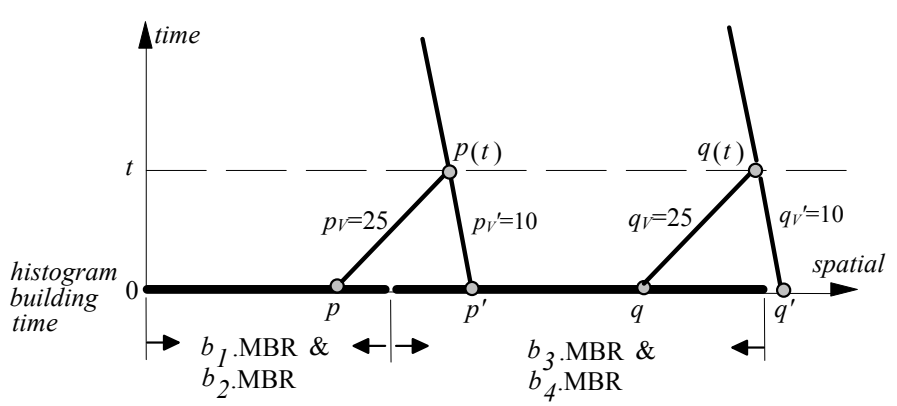

(b) Point $p$ falls in a different bucket after update

Figure 13: Updating the histogram 
An exhaustive scan over all buckets is necessary to identify the ones affected by an update. To avoid this, we build an in-memory ${ }^{4}$ TPR-tree on the MBRs and VBRs of the buckets (stored at the leaf level of the tree), so that the affected ones can be identified very quickly by performing a window query using the information of $p$ and $p^{\prime}$ respectively. Since, as with normal TPR-trees, the non-leaf levels account for a fraction of the total size, the space overhead is very small (less than $15 \%$ in our implementation). Similarly, building the tree after the histogram has been constructed requires less than $1 \%$ of the total construction time. Furthermore, notice that for most updates (except those requiring expansion) the bucket extents are not changed, and thus the TPR-tree only needs to be maintained infrequently.

Updating the histogram incrementally avoids the frequent histogram re-building, and hence reduces the maintenance cost considerably. Whenever the system receives an object update, the new information is intercepted to modify the histogram accordingly. However, the uniformity (in buckets) may gradually deteriorate along with time as the data (location and velocity) distributions vary. When the distribution changes significantly, the histogram eventually needs to be re-built in order to ensure satisfactory estimation accuracy. In order to formulate such a re-building condition, notice that, if sufficient distribution changes have accumulated, the histogram must have been modified many times. Therefore, a simple heuristic to ensure satisfactory estimation accuracy is to re-construct the histogram when the number of modifications reaches a certain threshold. As evaluated in the experiments, high prediction precision can be achieved with a very large threshold which leads to only occasional re-building.

\subsection{Performing Estimation with Histograms}

Given the histograms $H_{1}$ and $H_{2}$ for datasets $S_{1}$ and $S_{2}$ respectively, the expected number of qualifying join pairs can be obtained by summing up the results of bucket pairs from $H_{1} \times H_{2}$. Specifically, for two buckets $b_{i}, b_{j}\left(b_{i} \in H_{1}\right.$ and $\left.b_{j} \in H_{2}\right)$, the number of result pairs produced from objects inside them is $b_{i} \cdot n u m \cdot b_{j} . n u m \cdot \operatorname{Sel}_{i j}\left(d, t_{q}, m\right)$, where $\operatorname{Sel}_{i j}\left(d, t_{q}, m\right)$ is computed using equations 6 (for points), and 7 (for rectangles), by replacing $R_{i}, R_{j}, V_{i}, V_{j}$ with $b_{i}$. MBR, $b_{j}$.MBR, $b_{i}$.VBR, $b_{j}$.VBR respectively. Thus, the join selectivity can be estimated as:

$$
\operatorname{Sel}\left(d, t_{q}, m\right)=\frac{\sum_{\substack{\text { for all buckets } \\ b_{i} \in H_{1} \text { and } b_{j} \in H_{2}}} b_{i} \cdot \text { num } \cdot b_{j} \cdot \text { num } \cdot \operatorname{Sel}_{i j}\left(d, t_{q}, m\right)}{N_{1} \cdot N_{2}}
$$

where $N_{1}$ and $N_{2}$ are the cardinalities of $S_{1}$ and $S_{2}$ respectively.

\footnotetext{
${ }^{4}$ As currently there is no version of the TPR-tree specifically designed for main memory, our implementation follows the disk-resident version, although with a different node size $(=10)$. This choice of the node capacity is discussed towards the end of the section.
} 
As explained in the appendix, computing $\operatorname{Sel}_{i j}\left(d, t_{q}, m\right)$ requires integral evaluation, and hence should be avoided as much as possible in order to minimize the estimation time. For point data, given two buckets $b_{i}, b_{j}$, such computation is necessary only if the distance between $b_{i}\left(t_{q}\right)$ (i.e., its extent at query time $t_{q}$ ) and $b_{j}\left(t_{q}\right)$ is closer than the distance threshold $d$ (in the sequel we call $\left\langle b_{i}, b_{j}\right\rangle$ a qualifying bucket pair). Consider, for example, Figure 14 which shows the MBRs (at the current time) of 3 buckets: $b_{l} \in H_{l}$ and $b_{2}, b_{2}{ }^{\prime} \in H_{2}$, together with the VBRs. The dashed rectangles represent the extents of the buckets at query time $t_{q}$. Note that, computing $\operatorname{Sel}_{i j}\left(d, t_{q}, m\right)$ is necessary for $<b_{1}, b_{2}>\left(\left|b_{1}\left(t_{q}\right), b_{2}\left(t_{q}\right)\right|=0<d\right)$, but not for $<b_{1}, b_{2}{ }^{\prime}>$ because $\left|b_{1}\left(t_{q}\right), b_{2}{ }^{\prime}\left(t_{q}\right)\right|>d$, indicating that no points from $b_{1}$ and $b_{2}{ }^{\prime}$ can produce a joined pair. Similarly, for rectangular data, we do not consider two buckets if $\left|b_{1}\left(t_{q}\right), b_{2}\left(t_{q}\right)\right|>d+1 / 2\left(b_{1} . l e n+b_{2} . l e n\right)$, where $b_{1}$.len corresponds to the side length of the rectangles in $b_{1}$ (similar for $b_{2}$.len). This is because (i) $b_{1}$. MBR covers the centroids of the rectangles counted in $b_{1}$, and (ii) $\left|r_{1}, r_{2}\right| \leq d$ if and only if their centroids are closer than $d+1 / 2\left(r_{1}\right.$ len $+r_{2}$ len) along all dimensions (as explained in Section 3), where $r_{i}$ llen denotes the side length of $r_{i}$.

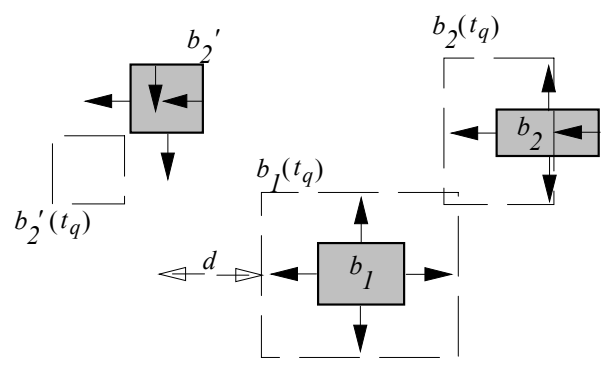

Figure 14: Buckets that need to be examined

In order to avoid checking all possible bucket pairs, we take advantage of the main-memory TPR-trees (used also for efficient updating of the histograms). Specifically, we adopt a synchronous traversal algorithm (i.e., SpatialJoin [5]) that traverses the TPR-trees of the two histograms synchronously, only following pairs of nodes that may lead to qualifying buckets. Compared with the brute-force approach, this algorithm avoids checking pairs whose parent entries do not qualify, thus leading to lower estimation time. Note that the node size influences the estimation time considerably. Specifically, a large size leads to a tree with few levels, in which case the intermediate entries have large MBRs and VBRs. Thus, the synchronous traversal needs to descend almost all pairs of (intermediate) entries, degenerating into the naïve algorithm (that checks all pairs of buckets). On the other hand, a small node size increases the number of tree levels, resulting in longer processing time on intermediate entries. In our experiments, we set the node size to 10 entries per node, which leads to the smallest traversal cost. 


\section{EXPERIMENTS}

In this section we present an extensive experimentation to prove the effectiveness of the proposed methods. All experiments were performed using a Pentium IV $1 \mathrm{GHz}$ CPU with 256 Mbytes memory. Due to the lack of real spatio-temporal data, we created synthetic datasets by generating velocities for objects in real spatial datasets. Figure 15 shows the distributions of the selected static datasets (the universe is normalized to $[0,10000])$, where $L B, C A$ contain point and $L A, G E R$ rectangle objects. From each dataset $X(=L B, C A, L A$, or $G E R)$, we created two spatio-temporal sets $X \_u$ and $X \_s$ where objects' velocities are generated according to uniform and Zipf distributions (with skew coefficient 0.8 ), respectively.

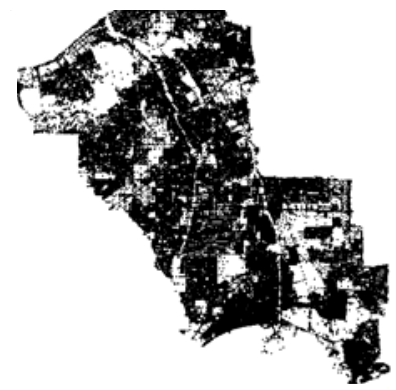

(a) $L B$ (53k points)

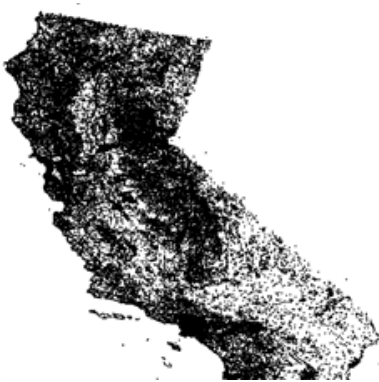

(b) $C A$ (62k points)

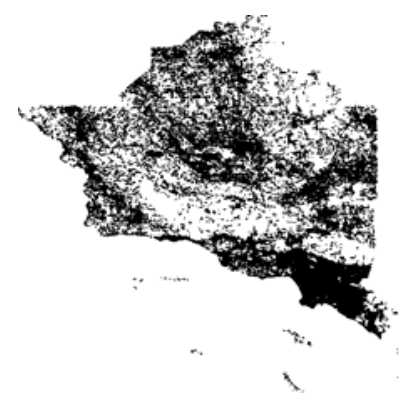

(c) $L A$ (130k rectangles)

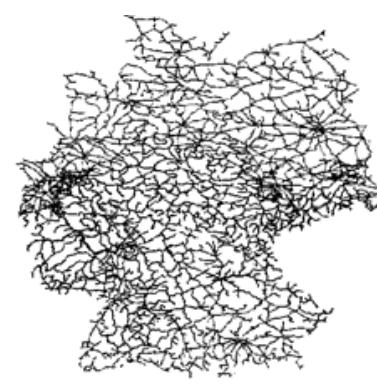

(d) GER (36k rectangles)

Figure 15: Location distributions

The accuracy of our model is examined using (i) the histogram in [7] where bucket extents are decided by considering only the spatial dimensions (referred to as 2D in the sequel), and (ii) the 4D histogram presented in Section 4. Both histograms are created using the MinSkew algorithm introduced in Section 2, while the resolution $H$ is set to 50 and 10 for $2 \mathrm{D}$ and $4 \mathrm{D}$ respectively (i.e., there are $50^{2}=2500$ (2D) and $10^{4}=10000$ (4D) cells in the initial grid before MinSkew starts). As shown in [2], the accuracy of Minskew initially improves as $H$ increases, but actually deteriorates when $H$ grows beyond a certain threshold. Values 50 and 10 (for 2D and 4D) are selected because they lead to the best performance for the corresponding histograms (in particular, 50 is also the value used in [7]). The number of buckets in a histogram is set to 200 (requiring around 8k bytes) in all cases. The 2D (4D) building time is around 0.5 (0.75) seconds after the initial grid is ready. A join query has two parameters: (i) the query timestamp $t_{q}$ (assuming the histogram is constructed at current time 0), and (ii) a distance threshold $d$. Given the actual $a c t$ and estimated est selectivity, the error rate is computed as $|e s t-a c t| / \min (e s t, a c t)$.

Figure 16a shows the estimation error for joining datasets $L B \_u$ and $C A \_u$ (i.e., uniform velocity distributions) by fixing the distance threshold $d$ to 250 and varying $t_{q}$ from 0 (i.e., the current time) to 500 timestamps in the future. Both histograms demonstrate similar behavior and provide precise estimation (with less than 5\% error). Note that, since the velocity distribution is uniform, the 4D histogram partitions mainly on the spatial dimensions, and thus behaves similarly to $2 \mathrm{D}$ (i.e., ignoring velocities is not 
important in this case). Figure $16 \mathrm{~b}$ illustrates the error rate as a function of $d$ with $t_{q}$ fixed to 250. Again both histograms have similar accuracy, indicating the correctness of the proposed equations. The error rates are relatively higher (around 10\%) when $d$ is small. This is not surprising because for low values of $d$ the output size is small, rendering accurate estimation more difficult.

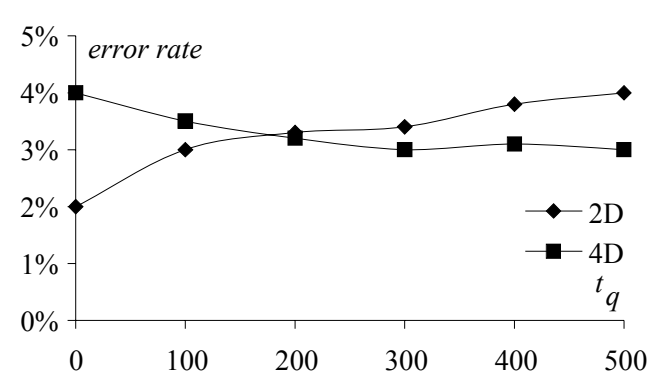

(a) Error vs. $t_{q}(d=250)$

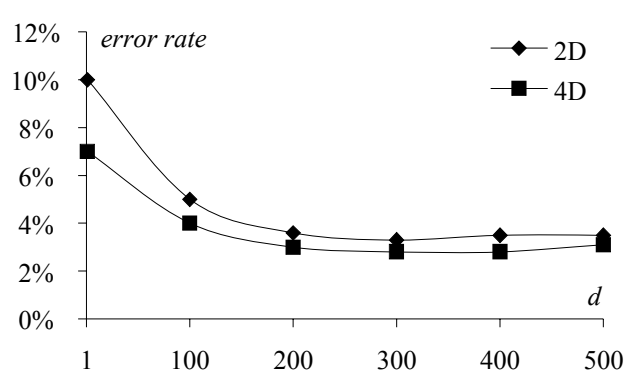

(b) Error vs. $d\left(t_{q}=250\right)$

Figure 16: $L B \_u$ joins $C A \_u$ (point data - uniform velocities)

Figure $17 \mathrm{a}$ and $\mathrm{b}$ demonstrate the results of similar experiments using datasets $L B \_s$ and $C A \_s$ (skewed velocity distribution). The performance of $2 \mathrm{D}$ is very poor in most cases (with up to $60 \%$ error) while the proposed 4D approach yields significantly lower error (up to 10\%). As shown in Figure 17a, 2D gives better estimation only when $t_{q}$ equals 0 (i.e., at the current time), while its precision deteriorates very quickly when $t_{q}$ increases. This is expected because the selectivity estimation at the current time depends on only objects' current location (i.e., not on velocities); thus, the 2D histogram is more accurate because it achieves better spatial uniformity in the buckets. 4D, on the other hand, outperforms it very quickly at small $t_{q}$, confirming the importance of considering velocities in building the histogram. Figure $17 \mathrm{~b}$ illustrates the error rate as a function of $d$ (fixing $t_{q}$ to 250). Similar to Figure 16b, the error rates remain stable when $d$ is sufficiently large.

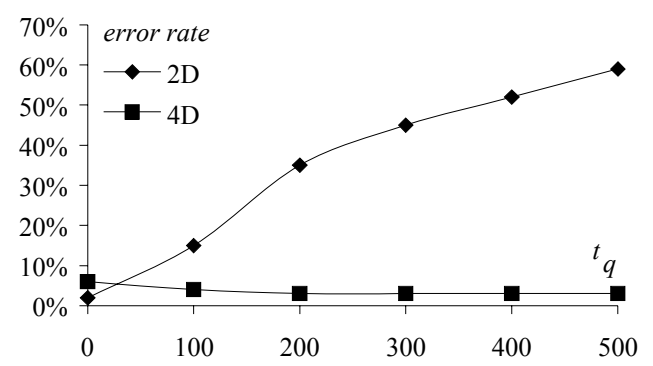

(a) Error vs. $t_{q}(d=250)$

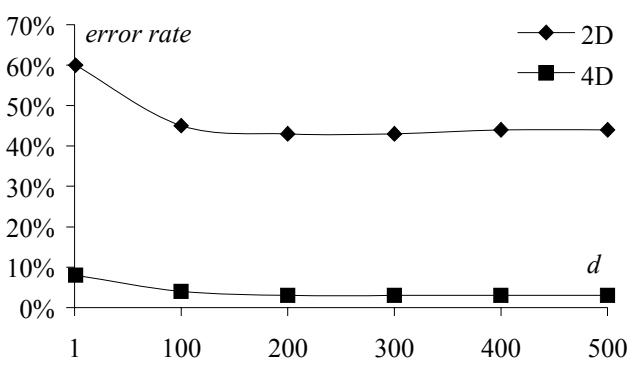

(b) Error vs. $d\left(t_{q}=250\right)$

Figure 17: $L B \_s$ joins $C A \_s$ (point data - skewed velocities)

Figure 18 and Figure 19 repeat the same set of experiments for rectangle data, where similar phenomena can be observed (again, 2D is erroneous for skewed velocities while 4D is accurate in all cases). Note that rectangles incur higher error than points because although rectangles have variable sizes, only the average size (in each bucket) is used for estimation. 


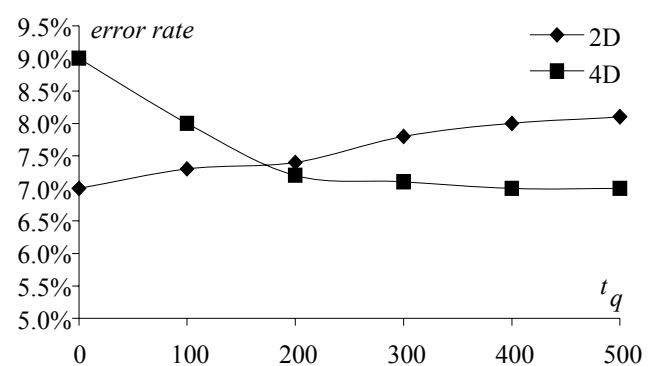

(a) Error vs. $t_{q}(d=250)$

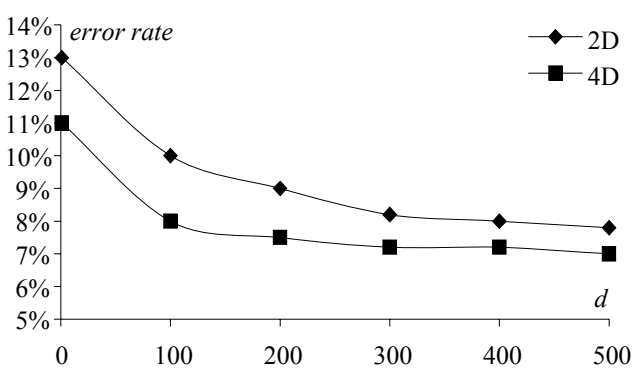

(b) Error vs. $d\left(t_{q}=250\right)$

Figure 18: $L A \_u$ joins $G E R \_u$ (rectangle data - uniform velocities)

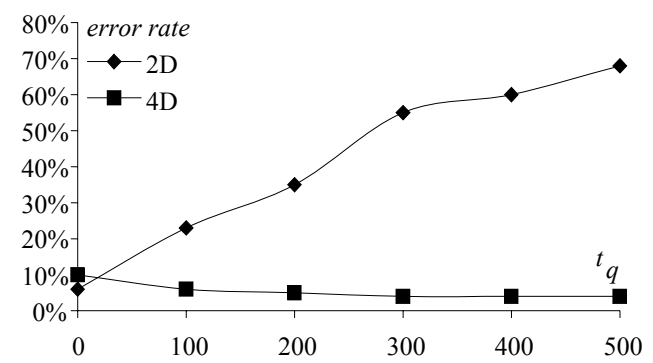

(a) Error vs. $t_{q}(d=250)$

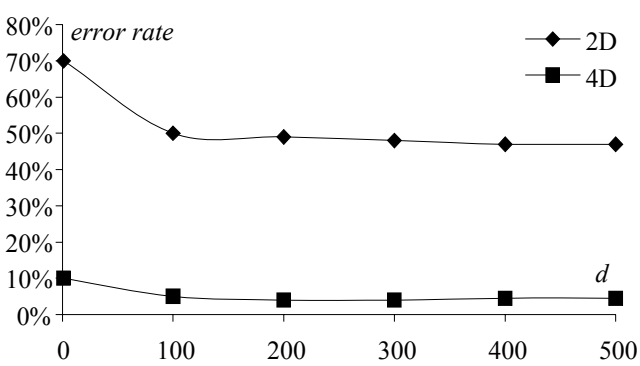

(b) Error vs. $d\left(t_{q}=250\right)$

Figure 19: $L A \_s$ joins $G E R \_s$ (rectangle data - skewed velocities)

The next experiment evaluates the efficiency of the cost models for constrained joins. Since the selectivity depends on the concrete position of the constraint window, we measure the average error of a workload consisting of 50 joins, where the constraint area of each join is a square uniformly distributed in the universe. Figure 20a and $\mathrm{b}$ demonstrate the results (obtained from equation 8) for point and rectangle datasets (with skewed velocities), respectively ( $d$ and $t_{q}$ are set to 250). The error rate is plotted as a function of the constraint window size, denoted as the percentage of its side length over the axis length (e.g., a $1 \%$ window covers $0.01 \%$ of the universe area). It is clear that $4 \mathrm{D}$ gives much more accurate prediction than $2 \mathrm{D}$. The error rates are relatively higher (up to $15 \%$ for $4 \mathrm{D}$ ) for small windows since they lead to high selectivity. On the other hand, when the window is sufficiently large, the error rate converges to that of a normal join.

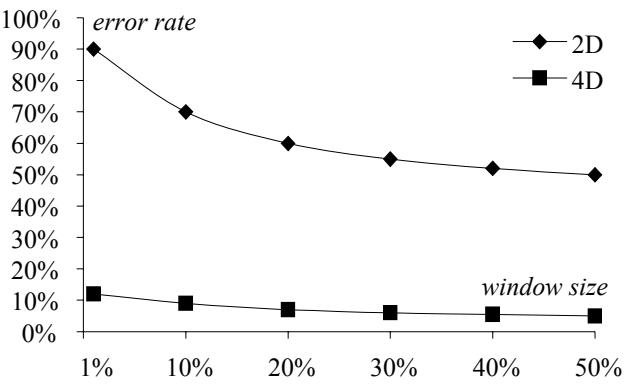

(a) $L B \_s-C A \_s$ (points - skewed velocities)

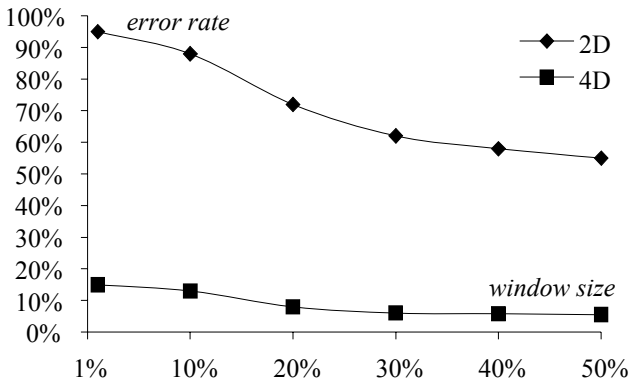

(b) $L A \_s-G E R \_s$ (rectangles - skewed velocities)

Figure 20: Error rates for constrained join $\left(d=250, t_{q}=250\right)$ 
As mentioned in Section 4.1, our histogram can be maintained incrementally to avoid frequent re-building. To study the effects of updates we created dynamic datasets as follows. At timestamp 0 , the location and velocities of objects are generated as described earlier. Then, at each of the subsequent 1000 timestamp, $10 \%$ of the objects are randomly chosen to produce updates. Specifically, the velocity change (along each dimension) is generated randomly in $[-10,10]$, while the new location of the object is decided based on its previous location and velocity. As discussed in Section 4.2, we build a main-memory TPR-tree on the histogram of each dataset, with a node capacity of 10 entries. The qualifying buckets are identified by synchronously traversing the two trees. The construction time for each tree is around $1 \%$ of the histogram building time. Further, the TPR-tree handles object updates efficiently in less than $0.1 \mathrm{~ms}$ per update.

Since the generated datasets incur (slow) distribution changes, whereas we do not modify the bucket extents, the uniformity inside each bucket may gradually deteriorate, thus affecting the estimation accuracy. To investigate the degradation rate, we measure the error of joins with the same parameters $\left(t_{q}=250, d=250\right)$ every 100 timestamps (i.e., based on objects' latest information) using the histogram incrementally maintained. Figure 21 shows the results (of the $4 \mathrm{D}$ histogram) for joining $L B \_s, C A \_s$ and $L A \_s, G E R \_s$. The accuracy decreases slowly with time (error rate up to $15 \%$ after 1000 timestamps). This implies that, using incremental maintenance we only need to re-build the histogram very infrequently in order to ensure good estimation accuracy. Note that similar experiments are not applicable to $2 \mathrm{D}$ histograms, which require re-building at every timestamp. This fact severely limits the applicability of $2 \mathrm{D}$ histograms in practice, where frequent updates may invalidate the histogram even before it is constructed.

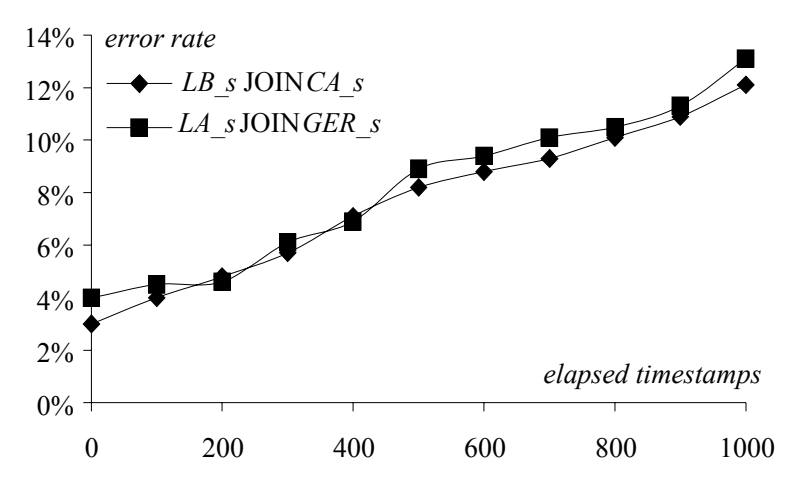

Figure 21: The accuracy degradation of 4D histogram $\left(t_{q}=250, d=250\right)$

As discussed in Section 4.2, given two histograms, a brute-force estimation algorithm would examine all pairs of buckets, and hence its computation time would be quadratic to the number of buckets. Instead we utilize the TPR-tree for each histogram and apply a spatial join algorithm to avoid examining unnecessary buckets. Figure 22 compares the evaluation time of the brute-force and TPR approaches in joining $L B \_s$ and $C A_{-} s$ (results for other datasets are similar). Specifically, Figure 22a shows the time as a function of 
$t_{q}$, fixing $d$ to 250. The cost of the brute force algorithm is constant because it checks the same number of bucket pairs for all queries. The cost of TPR, on the other hand, increases with time because, for larger $t_{q}$, the entry MBRs (at $t_{q}$ ) of the TPR-trees are larger, leading to more intersecting pairs. Figure $22 \mathrm{~b}$ shows similar results by varying $d$ and fixing $t_{q}$ to 250 (i.e., higher distance thresholds also increase the number of intersecting pairs).

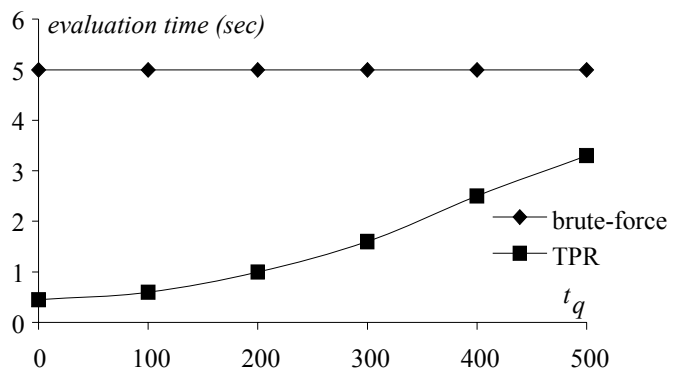

(a) Evaluation time vs. $t_{q}(d=250)$

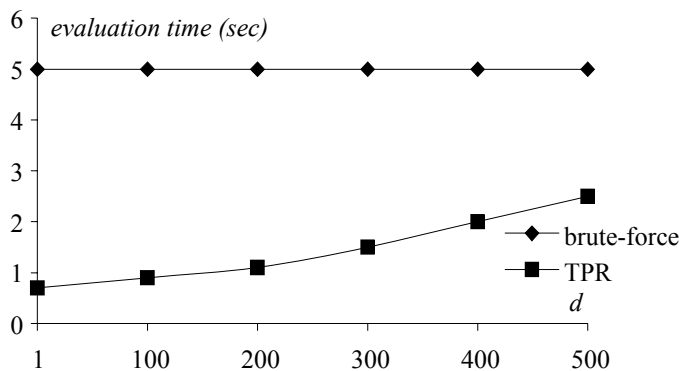

(b) Evaluation time vs. $d\left(t_{q}=250\right)$

Figure 22: Evaluation time $\left(L B \_s\right.$ join $\left.C A \_s\right)$

In summary, we have shown that the proposed models accurately capture the selectivity of spatiotemporal joins in a wide range of settings (including constrained joins), yielding average error below 10\%. Furthermore, we also confirm the necessity of considering velocity distributions in building spatiotemporal histograms, by demonstrating that the proposed 4D histogram has significantly lower error than the $2 \mathrm{D}$ counterpart.

\section{CONCLUSION}

The paper first discusses the mathematical preliminaries for spatio-temporal join selectivity and then solves the problem with a specialized histogram that can be efficiently maintained in main memory. Extensive experiments confirm that the proposed techniques produce accurate prediction with average error less than $10 \%$. Our techniques are based on the assumption that objects move with linear and known velocities, which is common in the literature of spatio-temporal prediction, e.g., [1, 7, 14, 23, 24]. Applications that satisfy these conditions involve objects (ships, airplanes, weather patterns) moving in unobstructed spaces. The prediction horizon depends on the (velocity) update rate; e.g., given that ships move with slow, linear movements for long periods, it is meaningful to estimate queries that refer to several hours in the future. For air traffic control, the prediction horizon should be in the order of minutes [26]. On the other hand, in some situations (e.g., cars on a road network) the motion parameters may be unknown or may change so fast that velocity-based prediction is meaningless. In such cases, selectivity estimation should be based on alternative techniques. For instance, [25] apply exponential smoothing using only location information. Although this method does not rely on velocity assumptions, it has to 
maintain historical data since future prediction is based on the recent past (whereas velocity-based prediction requires only information about the present).

\section{ACKNOWLEDGMENTS}

This was work was supported by grants HKUST 6180/03E and CityU 1163/04E from Hong Kong RGC.

\section{REFERENCES}

[1] P.K. Agarwal, L. Arge, J. Erickson, Indexing Moving Points. ACM PODS, 2000.

[2] S. Acharya, V. Poosala, S. Ramaswamy, Selectivity Estimation in Spatial Databases. ACM SIGMOD, 1999.

[3] W. Aref, H. Samet, A Cost Model for Query Optimization Using R-Trees. ACM GIS, 1994.

[4] N. An, Z. Yang, Sivasubramaniam, A. Selectivity Estimation for Spatial Joins. IEEE ICDE, 2001.

[5] T. Brinkhoff, H. Kriegel, B. Seeger, Efficient Processing of Spatial Joins Using R-trees. ACM SIGMOD, 1993.

[6] N. Beckmann, H. Kriegel, R. Schneider, B. Seeger, The R*-tree: An Efficient and Robust Access Method for Points and Rectangles. ACM SIGMOD, 1990.

[7] Y. Choi, C. Chung, Selectivity Estimation for Spatio-Temporal Queries to Moving Objects. ACM SIGMOD, 2002.

[8] A. Dobra, M. Garofalakis, J. Gehrke, R. Rastogi, Processing Complex Aggregate Queries over Data Streams. ACM SIGMOD, 2002.

[9] C. Faloutsos, B. Seeger, A. Traina, C. Traina Jr., Spatial Join Selectivity Using Power Laws. ACM SIGMOD, 2000.

[10] A. Guttman, R-Trees: A Dynamic Index Structure for Spatial Searching. ACM SIGMOD, 1984.

[11] J. Gehrke, F. Korn, D. Srivastava, On Computing Correlated Aggregates over Continual Data Streams. ACM SIGMOD, 2001.

[12] Y-W. Huang, N. Jing, E. Rundensteiner, Spatial Joins using R-trees: Breadth First Traversal with Global Optimizations. VLDB, 1997.

[13] I. Kamel, C. Faloutsos, On Packing R-Trees. CIKM, 1993.

[14] G. Kollios, D. Gunopulos, V. Tsotras, On Indexing Mobile Objects. ACM PODS, 1999.

[15] N. Koudas, K. Sevcik., High Dimensional Similarity Joins: Algorithms and Performance Evaluation. IEEE ICDE, 1998.

[16] N. Mamoulis, D. Papadias, Multiway spatial joins. ACM TODS, 26(4): 424-475, 2001.

[17] S. Muthukrishnan, V. Poosala, T. Suel, On Rectangular Partitionings in Two Dimensions: Algorithms, Complexity, and Applications. ICDT, 1999.

[18] W. Press, B. Flannery, S. Teukolsky, W. Vetterling, Numerical Recipes in C++ (second edition). Cambridge University Press, 2002.

[19] D. Pfoser, C. Jensen, Y. Theodoridis, Novel Approaches to the Indexing of Moving Object Trajectories. VLDB, 2000.

[20] B.U. Pagel, H.W. Six, H. Toben, P. Widmayer, Towards an Analysis of Range Query Performance in Spatial Data Structures. ACM PODS, 1993.

[21] D. Papadias, Y. Tao, P. Kalnis, J. Zhang, Indexing Spatio-Temporal Data Warehouses. IEEE ICDE, 2002.

[22] C. Sun, D. Agrawal, A. Abbadi, Selectivity Estimation for Spatial Joins with Geometric Selections. EDBT, 2002. 

ICDE, 2002.

[24] S. Saltenis, C.S. Jensen, S.T. Leutenegger, M.A. Lopez, Indexing the Positions of Continuously Moving Objects. ACM SIGMOD, 2000.

[25] J. Sun, D. Papadias, Y. Tao, B. Liu, Querying about the Past, the Present, and the Future in Spatio-Temporal Databases. IEEE ICDE, 2004.

[26] Y. Tao, J. Sun, D. Papadias, Analysis of Predictive Spatio-temporal Queries. ACM TODS, 28(4): 295-336, 2003.

[27] Y. Theodoridis, E. Stefanakis, T. Sellis, Cost Models for Join Queries in Spatial Databases. IEEE ICDE, 1998.

\section{APPENDIX}

This appendix discusses the evaluation of the integrals in equations 3 and 4 . For the sake of simplicity, we use the notation $\alpha=\left(l_{1}-l_{2}-d\right) / t_{q}$ and $\beta=\left(l_{1}-l_{2}+d\right) / t_{q}$; thus, the integral in equation 3 can be written as $\int_{V_{1}, v_{-}}^{V_{1} \cdot v_{+}} \int_{\max \left(V_{2} \cdot v_{-}, u_{1}+\alpha\right)}^{\min \left(V_{2}, v_{+}, u_{1}+\beta\right)} 1 d u_{2} d u_{1}$.

The value of the integral equals the area of the intersection (the shaded region in Figure 23a) of (i) the rectangle bounded by lines $\left\{u_{l}=V_{l} \cdot v_{-}, u_{l}=V_{l} \cdot v_{+}, u_{2}=V_{2} \cdot v_{-}, u_{2}=V_{l} \cdot v_{+}\right\}$, and (ii) the region between parallel lines $L N_{1}: u_{2}=u_{1}+\alpha$ and $L N_{2}: u_{2}=u_{1}+\beta$. As shown inFigure $23 \mathrm{~b}$ and $\mathrm{c}$, the shaded area is the difference of areas $A r_{l}$ and $A r_{2}$, i.e., the intersection of the rectangle in (i) and the lower half planes of lines $L N_{l}$ and $L N_{2}$ respectively. Obviously $A r_{1}$ depends on the intercept (i.e., $\alpha$ ) of $L N_{l}$, which decides the relative positions of $L N_{l}$ and the rectangle; Table A.1 illustrates its values for the all six possible cases. Due to the symmetry, values of $A r_{2}$ (as well as the corresponding conditions) are the same as those in Table 1, except that $\alpha$ should be replaced with $\beta$. Notice that since the integral in equation 3 equals $A r_{1}-A r_{2}$, we have solved the equation into closed form.

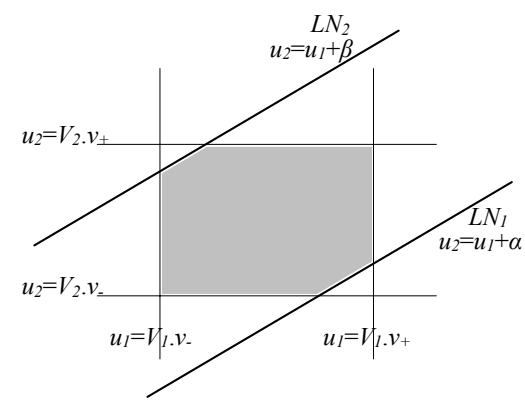

(a) Integral area

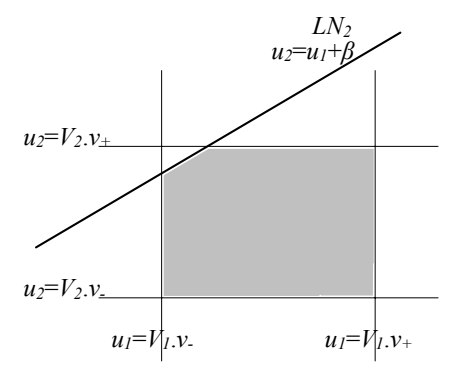

(b) Area $A r_{1}$

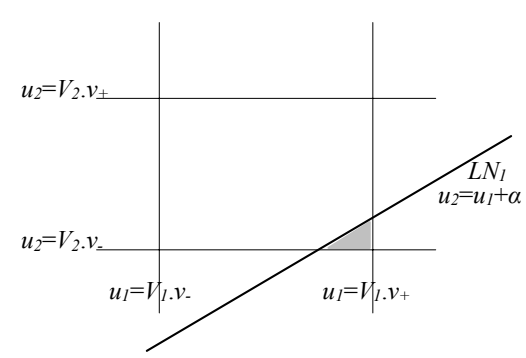

(c) Area $\mathrm{Ar}_{2}$

Figure 23: Integral area $=A r_{1}-A r_{2}$

Because the exact solution of equation 4 is more complex, in our implementation we evaluate it using the trapezoidal method [18]. Particularly, the numerical approach is applied to the two outer integral layers 
(recall that equation 4 has four layers), while the inner two (i.e., corresponding to equation 3) can be solved accurately as described earlier.

\begin{tabular}{|c|c|}
\hline Values of $A r_{1}$ & Conditions \\
\hline$\left(V_{1} \cdot v_{+}-V_{1} \cdot v_{-}\right)\left(V_{2} \cdot v_{+}-V_{2} \cdot v_{-}\right)$ & $\alpha \in\left(V_{2 .} v_{+}-V_{1 .} v_{-}, \infty\right)$ \\
\hline $\begin{array}{l}\frac{1}{2}\left[\left(V_{1} \cdot v_{-}-V_{2 .} v_{-}+\alpha\right)+\left(V_{2 . v+}-V_{2 .} v_{-}\right)\right]\left(V_{2 .} v_{+}-V_{1 .} v_{-}-\alpha\right) \\
+\left(V_{1} . v_{+}-V_{2} . v_{+}+\alpha\right)\left(V_{2 .} v_{+}-V_{2} . v_{-}\right)\end{array}$ & $\alpha \in\left(\begin{array}{l}\max \left\{V_{2 . v-}-V_{1} \cdot v_{-}, V_{2} . v_{+}-V_{1} . v+\right\} \\
V_{2 . v+}-V_{1 . v} v_{-}\end{array}\right]$ \\
\hline$\frac{1}{2}\left[\left(V_{1} \cdot v_{+}-V_{2} \cdot v_{+}+\alpha\right)+\left(V_{2} \cdot v_{+}-V_{2} \cdot v_{-}+\alpha\right)\right]\left(V_{2} \cdot v_{+}-V_{2 . v} \cdot\right)$ & $\alpha \in\left(V_{2 . v_{+}}-V_{1 . v_{+},} V_{2 . v_{-}}-V_{1 . v_{-}}\right]$ \\
\hline$\frac{1}{2}\left[\left(V_{1} \cdot v_{-}-V_{2} \cdot v_{-}+\alpha\right)+\left(V_{1} \cdot v_{+}-V_{2} \cdot v_{-}+\alpha\right)\right]\left(V_{1} \cdot v_{+}-V_{1} \cdot v_{-}\right)$ & $\alpha \in\left(V_{2 . v-v_{-}}-V_{1 .} v_{-}, V_{2 . v+}-V_{1 . v_{+}}\right]$ \\
\hline$\frac{1}{2}\left(V_{1} . v_{+}-V_{2 . v-}+\alpha\right)\left(V_{1} . v_{+}-V_{2 . v-}+\alpha\right)$ & $\alpha \in\left(\begin{array}{l}V_{2} . v_{-}-V_{1} . v_{+} \\
\min \left\{V_{2} . v_{-}-V_{1} . v_{-}, V_{2} . v_{+}-V_{1} . v_{+}\right\}\end{array}\right]$ \\
\hline 0 & $\alpha \in\left(-\infty, V_{2 .} v_{-}-V_{1 . v+}\right]$ \\
\hline
\end{tabular}

Table 2: Values of $A r_{1}$ and corresponding conditions 